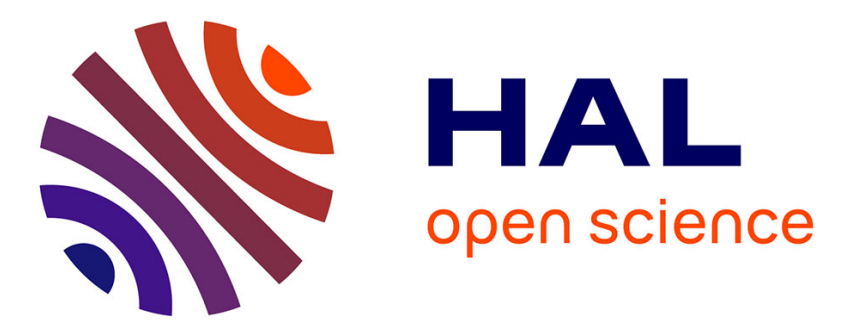

\title{
Efficient and Accurate Algorithm for the Full Modal Green's Kernel of the Scalar Wave Equation in Helioseismology
}

Hélène Barucq, Florian Faucher, Damien Fournier, Laurent Gizon, Ha Pham

\section{- To cite this version:}

Hélène Barucq, Florian Faucher, Damien Fournier, Laurent Gizon, Ha Pham. Efficient and Accurate Algorithm for the Full Modal Green's Kernel of the Scalar Wave Equation in Helioseismology. SIAM Journal on Applied Mathematics, 2020, 80 (6), pp.2657-2683. 10.1137/20M1336709 . hal-03101642

\author{
HAL Id: hal-03101642 \\ https://hal.science/hal-03101642
}

Submitted on 15 Feb 2021

HAL is a multi-disciplinary open access archive for the deposit and dissemination of scientific research documents, whether they are published or not. The documents may come from teaching and research institutions in France or abroad, or from public or private research centers.
L'archive ouverte pluridisciplinaire HAL, est destinée au dépôt et à la diffusion de documents scientifiques de niveau recherche, publiés ou non, émanant des établissements d'enseignement et de recherche français ou étrangers, des laboratoires publics ou privés. 


\title{
EFFICIENT AND ACCURATE ALGORITHM FOR THE FULL MODAL GREEN'S KERNEL OF THE SCALAR WAVE EQUATION IN HELIOSEISMOLOGY*
}

\author{
HÉLÈNE BARUCQ ${ }^{\dagger}$, FLORIAN FAUCHER $^{\ddagger}$, DAMIEN FOURNIER $^{\S}$, LAURENT GIZON $^{\S}$, \\ AND HA PHAM ${ }^{\dagger}$
}

Abstract.

In this work, we provide an algorithm to compute efficiently and accurately the full outgoing modal Green's kernel for the scalar wave equation in local helioseismology under spherical symmetry. Due to the high computational cost of a full Green's function, current helioseismic studies only use its values at a single depth. However, a more realistic modelisation of the helioseismic products (cross-covariance and power spectrum) requires the full Green's kernel. In the classical approach, the Dirac source is discretized and one simulation gives the Green's function on a line. Here, we propose a two-step algorithm which, with two simulations, provides the full kernel on the domain. Moreover, our method is more accurate as the singularity of the solution due to the Dirac source is described exactly. In addition, it is coupled with the exact Dirichlet-to-Neumann boundary condition, providing optimal accuracy in approximating the outgoing Green's kernel, which we demonstrate in our experiments. In addition, we show that high-frequency approximations of the nonlocal radiation boundary conditions can represent accurately the helioseismic products.

Key words. modal Green's kernel, helioseismology, radiation boundary conditions, helioseismic observables, Whittaker's functions, hybridizable discontinuous Galerkin

AMS subject classifications. 34B27, 33C15, 65N80, 85-04, 85-08, 35J10, 35L05, 35A08

1. Introduction. In this work, we propose an efficient algorithm to compute accurately the full outgoing modal Green's kernel for the scalar wave equation in local helioseismology under spherical symmetry. The outgoing Green's kernel, denoted by $\mathcal{G}(\mathbf{x}, \mathbf{y})$, is the key ingredient in obtaining simulated observables which are then used in data analysis in time-distance helioseismology, cf. section 5 . At the angular frequency $\omega, \mathcal{G}$ is a solution to, $[5,8]$,

$$
-\nabla \cdot\left(\frac{1}{\rho(\mathbf{x})} \nabla \mathcal{G}(\mathbf{x}, \mathbf{y})\right)-\frac{\sigma^{2}(\mathbf{x}, \omega)}{\rho(\mathbf{x}) \mathrm{c}^{2}(\mathbf{x})} \mathcal{G}(\mathbf{x}, \mathbf{y})=\delta(\mathbf{x}-\mathbf{y})
$$

where the background of the Sun is characterized by the density $\rho$ and the sound speed c. Here, $\delta$ is the Dirac function and $\sigma$ denotes the complex frequency that contains a model of attenuation. In other applications, (1.1) is also called the Bergmann's equation ([9]) for inhomogeneous acoustic media, cf. [24, Section II.A]. In helioseismology, it is obtained from the equation for the wave displacement $\boldsymbol{\xi}$ without background flow and rotation, cf., e.g., [23],

$$
-\frac{1}{\rho} \nabla\left(\rho \mathrm{c}^{2} \nabla \cdot \boldsymbol{\xi}\right)-\sigma^{2} \boldsymbol{\xi}+\text { gravity terms }=\boldsymbol{s} .
$$

By taking the divergence on both side of (1.2), considering the scalar quantity $\rho c^{2} \nabla \cdot \boldsymbol{\xi}$ as unknown, and if we neglect gravity and the gradient of $\sigma$, we obtain the scalar

\footnotetext{
*Submitted to the editors February 15, 2021.

$\dagger$ Inria Project-Team Magique 3D, E2S-UPPA, CNRS, Pau, France (helene.barucq@inria.fr, ha.howard@inria.fr).

${ }^{\ddagger}$ Faculty of Mathematics, University of Vienna, Oskar-Morgenstern-Platz 1, A-1090 Vienna, Austria (florian.faucher@univie.ac.at).

§Max-Planck-Institut für Sonnensystemforschung, Justus-von-Liebig-Weg 3, 37077 Göttingen, Germany (fournier@mps.mpg.de, gizon@mps.mpg.de).
} 
operator in (1.1), cf. [17, Section 2]. Due to the omission of the gravity (necessary for the reduction to a scalar problem), equation (1.1) does not take into account buoyancy, and cannot be used to model internal and surface gravity waves. However, it still allows for a good description of acoustic modes and simulates correctly the observed solar data, cf. [17].

Similarly to $[8,7,1]$, we work with the conjugated equation obtained by a Liouville change of unknowns, since the latter offers a natural setting to define the unique physical (also called "outgoing" or "radiation") kernel $G$, solution to, [8],

$$
\left(-\Delta-\frac{\sigma^{2}(\mathbf{x}, \omega)}{\mathrm{c}^{2}(\mathbf{x})}+\mathrm{q}(\mathbf{x})\right) G(\mathbf{x}, \mathbf{y})=\delta(\mathbf{x}-\mathbf{y}), \quad \text { with } \mathbf{q}:=\rho^{1 / 2} \Delta \rho^{-1 / 2} .
$$

The Liouville transform is also discussed in [25, Eq. (2.9)]. In [24, 25], the difference is that the density and the sound speed are considered constant outside of the domain, so this is equivalent to a compact perturbation of the Helmholtz operator. In our case, we have a long-range perturbation and the density is assumed to be exponentially decaying outside of domain, following the solar Atmo model, [5]. From $G$, the physical Green's kernel for the original problem (1.1) is defined by

$$
\mathcal{G}(\mathbf{x}, \mathbf{y}):=\rho^{1 / 2}(\mathbf{x}) \rho^{1 / 2}(\mathbf{y}) G(\mathbf{x}, \mathbf{y}) .
$$

Assuming that the physical parameters only depend on the Sun's radius, one can decompose $G$ into the spherical harmonic basis, and compute instead the modal Green's kernel $G_{\ell}(r, s)$ for each spherical mode $\ell$, with $r=|\mathbf{x}|$ the (scaled) radius and $s$ the source position. It is a fundamental solution of, [8],

$$
\left(-\frac{d^{2}}{d r^{2}}-\frac{\sigma^{2}(r)}{\mathrm{c}^{2}(r)}+\mathrm{q}(r)+\frac{\ell(\ell+1)}{r^{2}}\right) G_{\ell}=\delta(r-s) .
$$

Current results in local helioseismology assume that the Sun has a "surface" that is well defined, and work with numerical simulations which are obtained from the solution of the wave equation at the surface of the Sun only, i.e., $G_{\ell}(r, s=1)$ using scaled variables. However, the Sun is a plasma and the observed oscillations represent an average over all depths weighted by the level of transparency (opacity). Therefore, the accurate computations of helioseismic products require the full Green's function. In addition, it is also required to interpret the multi-height HMI (Helioseismic and Magnetic Imager) data [26] and to image the solar interior using helioseismic holography, [29].

Numerically, the current approach to evaluate the Green's function is to directly discretize (1.5) using, e.g., a finite element or a finite difference method, cf. [17, 5, 11]. Since each resolution only gives the value for a fixed source, it is expensive to obtain the "complete" Green's kernel which requires an arbitrarily high number of sources. Additionally, unlike in applications such as seismology or inverse scattering in which one is mostly interested in the far-field or the backscattered data obtained away from the source, in helioseismology, values at the same height of the source are particularly important. The presence of the Dirac however reduces the numerical accuracy of the response in the region around the source. To overcome this problem, one usually refines the mesh around the source, leading to additional computational cost, cf. $[11,16,5]$. With solar background, it is particularly expensive, since this extrarefinement comes in addition to the one needed to capture the profile of the model parameters. In our work, our algorithm provides, with only two simulations, the 
full Green's function, for each spherical mode $\ell$ and frequency $\omega$, without any extrarefinement.

The procedure we propose is based on a formula for Green's kernel in ODE theory and comprises of two steps. One first obtains solutions of two boundary value problems: one regular solution in a neighborhood of the origin and an outgoing one in an exterior neighborhood. They are then 'assembled' by a Heaviside function to give the global modal Green's kernel. The importance here is that while these solutions are regular, the singularity of the Green's kernel is captured exactly by the Heaviside function. In addition, working with regular solutions removes the need to refine the mesh around the source position. Namely, with only two problems, one instantly obtains the value of the modal Green's kernel on a rectangular region away from the origin, and thus of the 3D Green's kernel on a product of two punctured spheres.

Other novelties of our work are in the numerical implementation and computation of the outgoing Green's kernel, with the following three aspects.

1. The algorithm is implemented with Hybridizable Discontinuous Galerkin (HDG) discretization, [13, 20, 6], which, with unknowns being the numerical trace (boundary values), reduces the computational effort compared to other scheme.

2. We compute synthetic helioseismic products (power spectrum) using two atmospheric models: S+Atmo $([12,5])$ and S+Val-C ([28]), and compare several formation heights. Here, our work is the first to use the exact Dirichlet-to-Neumann $(\mathrm{DtN})$ coefficient for the radiation condition.

3. We investigate the efficiency of approximate radiation boundary conditions (RBC) on synthetic helioseismic products using the complete solar background. Until now, these comparisons were only carried out in terms of solutions in the atmosphere (e.g., [8]). With the exact DtN coefficient as reference, our investigation provides an efficient choice of approximate $\mathrm{RBC}\left(\mathbf{Z}_{\mathrm{S}-\mathrm{HF}-1 \mathrm{a}}\right.$, see section 5$)$, which is independent of the mode $\ell$. This is particularly useful for discretization schemes in $3 \mathrm{D}$ since, being independent of $\ell$, it does not contain tangential differential operators.

The article is organized as follows. In section 2, we formulate the problem with the appropriate boundary conditions, and state, in Proposition 2.4, the main result to construct the Green's function. We provide in section 3 the computational steps and validate our implementation comparing with analytical solutions to illustrate the efficiency of our approach. The helioseismic products are defined in section 4 and computed in section 5, illustrating the necessity of using the full Green's kernel.

2. Formulation of the solar Green's functions. In this section, we provide the dimensionless modal equation with the appropriate boundary conditions, and give the two-step strategy for the efficient computation of the Green's kernel via Proposition 2.4.

2.1. Problem with dimensionless coefficients. We consider the $3 \mathrm{D}$ coordinate system with the center of the Sun placed at the origin, and denote by $\check{\mathbf{x}}$ the position in this system. Our first task is to derive the adimensional version of the problem: with $R_{\odot}$ denoting the radius of the Sun, $\left(R_{\odot} \simeq 695.5 \times 10^{6} \mathrm{~m}\right)$, we introduce the scaled coordinates,

$$
\mathbf{x}=\frac{\check{\mathbf{x}}}{R_{\odot}}, \quad \text { such that } \quad \nabla_{\mathbf{x}}=R_{\odot} \nabla_{\check{\mathbf{x}}} .
$$

We introduce the scaled radius $r=R / R_{\odot}$, which is dimensionless, where $R=|\check{\mathbf{x}}|$.

The associated density function $\rho$, the dimensionless inverse scale height $\alpha$ and 
the scaled velocity $\mathfrak{c}$ are defined by

$$
r \mapsto \rho(r)=\rho\left(R_{\odot} r\right), \quad \alpha(r):=-\frac{\partial_{r} \rho(r)}{\rho(r)}, \quad \mathfrak{c}:=\frac{\mathrm{c}}{R_{\odot}} .
$$

Since $\partial_{r}=R_{\odot} \partial_{R}$, we have that $\alpha=\alpha / R_{\odot}$. Here, $\mathfrak{c}$ is expressed in $\mathrm{s}^{-1}$, so that the quantity $\sigma / \mathfrak{c}$ is dimensionless.

The main equation for the original problem is given by (1.1) and we have, for a generic right-hand side $\check{\mathfrak{f}}$,

$$
-\nabla \cdot\left(\frac{1}{\rho} \nabla \check{\mathfrak{u}}\right)-\frac{\sigma^{2}}{\rho \mathrm{c}^{2}} \check{\mathfrak{u}}=\check{\mathfrak{f}}, \quad \text { with } \quad \sigma^{2}(\mathbf{x}, \omega)=\omega^{2}+2 \mathrm{i} \omega \gamma(\mathbf{x}, \omega) .
$$

We recall that $\omega>0$ is the angular frequency, and $\gamma$ the attenuation. We first write the field $\check{\mathfrak{u}}$ and the right-hand side $\breve{f}$ in terms of the scaled radius:

$$
\mathfrak{u}(\mathbf{x}):=\check{\mathfrak{u}}\left(R_{\odot} \mathbf{x}\right), \quad \mathfrak{f}(\mathbf{x}):=\check{\mathfrak{f}}\left(R_{\odot} \mathbf{x}\right) .
$$

They satisfy the equation, using (2.1) and multiplying by $R_{\odot}^{2}$,

$$
-\nabla \cdot\left(\frac{1}{\rho} \nabla \mathfrak{u}\right)-\frac{\sigma^{2}}{\rho \mathfrak{c}^{2}} \mathfrak{u}=R_{\odot}^{2} \mathfrak{f} .
$$

The conjugated problem introduces the change of unknown, $[8,1]$,

$$
u(\mathbf{x})=\rho(\mathbf{x})^{-1 / 2} \mathfrak{u}(\mathbf{x}) .
$$

The dimensionless potential q for the conjugated problem is

$$
\mathrm{q}(\mathbf{x}):=\rho(\mathbf{x})^{1 / 2} \Delta_{\mathbf{x}} \rho(\mathbf{x})^{-1 / 2} .
$$

The conjugated equation with dimensionless coefficients writes as

$$
-\Delta_{\mathbf{x}} u-\frac{\sigma^{2}}{\mathfrak{c}^{2}} u+\mathrm{q} u=g, \quad \text { with } \quad g=\rho^{1 / 2} R_{\odot}^{2} \mathfrak{f} .
$$

2.2. Solar modal Green's function. Similarly to [8], we work with the conjugated problem, given by (2.8) in three dimensions after adimensionalization. Then, using spherical symmetry, we decompose the solution into one-dimensional modal ones defined on $\left[0, r_{\max }\right]$, with $r_{\max }>1$. Note that in the adimensionalized version, the position $r=1$ corresponds with the Sun's "surface" (at $R_{\odot}$ ). In terms of boundary conditions, a Neumann-like one holds at 0 , cf. subsection 2.3, while a radiation boundary condition is imposed at $r_{\max },[5,8]$.

We have denoted by $\mathcal{G}$ the Green's function of the original problem (cf. (1.1)), and that of the conjugated problem by $G$, which satisfies,

$$
\left(-\Delta_{\mathbf{x}}-\frac{\sigma^{2}(r)}{\mathfrak{c}^{2}(r)}+\mathrm{q}(r)\right) G(\mathbf{x}, \mathbf{s})=\delta(\mathbf{x}-\mathbf{s}),
$$

with

$$
\mathrm{q}(r)=\frac{\alpha^{2}(r)}{4}+\frac{\alpha^{\prime}(r)}{2}+\frac{\alpha(r)}{r} .
$$


Remark 2.1. In helioseismolgy, one usually writes the zero-th order term as

$$
\frac{\sigma^{2}}{\mathfrak{c}^{2}}-\mathrm{q}=\frac{\sigma^{2}-\omega_{c}^{2}}{\mathfrak{c}^{2}}
$$

where $\omega_{c}$ is called the local cut-off frequency:

$$
\omega_{c}^{2}(r):=\mathfrak{c}^{2}(r) \mathrm{q}(r)=\mathfrak{c}^{2}(r)\left(\frac{\alpha^{2}(r)}{4}+\frac{\alpha^{\prime}(r)}{2}+\frac{\alpha(r)}{r}\right) .
$$

This is the same expression as in [16, Eq. (11) and Figure 3], where it is shown that, despite the simplification of the wave equation (ignoring buoyancy), the cut-off frequency is consistent with solar applications, in particular its value at the beginning of the atmosphere is around $5.2 \mathrm{mHz}$. The sign of $\omega^{2}-\omega_{c}^{2}$ determines the local behaviour of the solution and waves with frequencies $\omega>\omega_{c}$ propagate into the atmosphere, cf. [16].

As mentionned in the introduction, the Green's functions are related by

$$
\mathcal{G}(\mathbf{x}, \mathbf{y})=\rho^{1 / 2}(\mathbf{x}) \rho^{1 / 2}(\mathbf{y}) G(\mathbf{x}, \mathbf{y}) .
$$

For more details on the transformation, we refer to $[8,1]$ and $[7$, Appendix A].

Assumption 2.2. The sound speed $\mathfrak{c}$ and attenuation coefficient $\gamma$ are bounded functions, which are constant outside of a compact set with $\mathfrak{c}_{\infty}$ and $\gamma_{\infty}$ denoting their respective values on the exterior domain. In another word, we have,

$$
\operatorname{Supp}\left(\mathfrak{c}-\mathfrak{c}_{\infty}\right) \text { is compact and } \operatorname{Supp}\left(\gamma-\gamma_{\infty}\right) \text { is compact. }
$$

Assumption 2.3. We assume that the background density $\rho$ is such that $\alpha=$ $-\rho^{\prime} / \rho$ satisfies, for constants $\alpha_{\infty}>0$ and $\epsilon>0$,

$$
\begin{aligned}
\alpha(r) & \in \mathcal{C}^{1}\left(\mathbb{R}_{+}\right) \cap L^{\infty}\left(\mathbb{R}_{+}\right), \quad \lim _{r \rightarrow \infty} \alpha=\alpha_{\infty}, \\
\text { and } \quad \alpha^{\prime}(r) & =\mathrm{O}(1+r)^{-(1+\epsilon)}, \quad r \rightarrow \infty
\end{aligned}
$$

Let us note that these assumptions are not particularly restrictive and, in particular, the solar velocity and density models S+Atmo ([12]) or S+Val-C ([28]) satisfy them. Under Assumption 2.3, the potential q has a finite limiting value denoted by $q_{\infty}$, such that

$$
\lim _{r \rightarrow \infty} \mathrm{q}=\mathrm{q}_{\infty}=\frac{\alpha_{\infty}^{2}}{4}
$$

and, as $r$ tends to 0 ,

$$
\lim _{r \rightarrow 0} r^{2}\left(-\frac{\sigma^{2}(r)}{\mathfrak{c}^{2}(r)}+\mathrm{q}(r)\right)=0 .
$$

Under Assumptions 2.2 and 2.3, we can apply the result of [2, Theorem 6.2] to obtain the physical Green's kernel. To state the proposition, we introduce the potential $Q_{\ell}$ such that,

$$
\frac{Q_{\ell}(r)}{r^{2}}=-\frac{\sigma^{2}(r)}{\mathfrak{c}^{2}(r)}+\mathrm{q}(r)+\frac{\ell(\ell+1)}{r^{2}}=-\frac{\sigma^{2}(r)}{\mathfrak{c}^{2}(r)}+\frac{\alpha^{2}(r)}{4}+\frac{\alpha^{\prime}(r)}{2}+\frac{\alpha(r)}{r}+\frac{\ell(\ell+1)}{r^{2}}
$$


and the operator $\mathrm{L}_{\ell}$,

$$
\mathrm{L}_{\ell}:=-\frac{d^{2}}{d r^{2}}+\frac{Q_{\ell}(r)}{r^{2}}=-\frac{d^{2}}{d r^{2}}-\frac{\sigma^{2}(r)}{\mathfrak{c}^{2}(r)}+\mathrm{q}(r)+\frac{\ell(\ell+1)}{r^{2}} .
$$

Furthermore, we denote by $\mathrm{Y}_{\ell}^{m}$ the $m$-th spherical harmonic of order $\ell$, by $\mathrm{P}_{\ell}$ the Legendre polynomial of degree $\ell, \mathrm{H}$ is the Heaviside function and the Wronskian is denoted $\mathcal{W}(s):=\mathcal{W}\{\psi(s), \tilde{\psi}(s)\}$. Following $[8,7]$, with $\sqrt{\cdot}$ using the argument branch $[0,2 \pi)$, we also introduce the conjugate wavenumber $k$ defined by

$$
\mathrm{k}=\sqrt{\frac{\sigma^{2}}{\mathfrak{c}_{\infty}^{2}}-\frac{\alpha_{\infty}^{2}}{4}} .
$$

Proposition 2.4 (Green's kernel expansion). Under Assumptions 2.2 and 2.3, the outgoing Green's function can be written as an expansion in spherical harmonic basis with, using - for the complex conjugation,

$$
\begin{aligned}
G(\mathbf{x}, \mathbf{y}) & =\frac{1}{|\mathbf{x}||\mathbf{y}|} \sum_{\ell=0}^{\infty} \sum_{m=-\ell}^{\ell} G_{\ell}^{m}(|\mathbf{x}|,|\mathbf{y}|) \mathrm{Y}_{\ell}^{m}(\hat{\mathbf{y}}) \overline{\mathrm{Y}_{\ell}^{m}(\hat{\mathbf{x}})}, \quad \mathbf{x}, \mathbf{y} \text { not on the z-axis } \\
& =\frac{1}{4 \pi|\mathbf{x}||\mathbf{y}|} \sum_{\ell=0}^{\infty}(2 \ell+1) G_{\ell}(|\mathbf{x}|,|\mathbf{y}|) \mathrm{P}_{\ell}(\hat{\mathbf{x}} \cdot \hat{\mathbf{y}}) .
\end{aligned}
$$

Here, $G_{\ell}^{m}(r, s)=G_{\ell}$ is independent of $m$ and is the unique distributional solution to

$$
\mathrm{L}_{\ell} G_{\ell}=\delta(r-s),
$$

satisfying the boundary condition,

$$
\lim _{r \rightarrow 0} r^{-(\ell+1)} G_{\ell}(r)=1,
$$

at $r=0$, and the asymptotic relation at infinity,

$$
G_{\ell}=e^{\mathrm{i} \varphi(r, \mathrm{k})}(1+\mathrm{o}(1)) \quad \text { as } r \rightarrow \infty .
$$

In (2.24), the phase function is defined for some $r_{0}>0$ as,

$$
\varphi(r):=\int_{r_{0}}^{r} \sqrt{\mathrm{k}^{2}-\frac{\alpha}{s}} \mathrm{~d} s=\mathrm{k} r-\frac{\alpha}{2 \mathrm{k}} \log r+\mathrm{k}^{-2} \mathrm{o}(1) .
$$

Furthermore, if $\tilde{\psi}_{\ell}$ and $\psi_{\ell}$ are two homogeneous solutions to $\mathrm{L}_{\ell} w=0$ on $(0, s)$ and $(s, \infty)$ respectively, with $\tilde{\psi}_{\ell}$ satisfying the boundary condition $(2.23)$ at $r=0$, and $\psi_{\ell}$ the condition (2.24) as $r \rightarrow \infty$, we have

$$
G_{\ell}(r, s)=\frac{-\mathrm{H}(s-r) \psi(r) \tilde{\psi}(s)-\mathrm{H}(r-s) \tilde{\psi}(r) \psi(s)}{\mathcal{W}(s)} .
$$

Remark 2.5. The kernel for the original equation is

$$
\begin{gathered}
\mathcal{G}(\mathbf{x}, \mathbf{y})=\frac{1}{4 \pi|\mathbf{x}||\mathbf{y}|} \sum_{\ell=0}^{\infty}(2 \ell+1) \mathcal{G}_{\ell}(|\mathbf{x}|,|\mathbf{y}|) \mathrm{P}_{\ell}(\hat{\mathbf{x}} \cdot \hat{\mathbf{y}}) \\
\text { where } \quad \mathcal{G}_{\ell}(|\mathbf{x}|,|\mathbf{y}|)=\rho^{1 / 2}(|\mathbf{x}|) \rho^{1 / 2}(|\mathbf{y}|) G_{\ell}(|\mathbf{x}|,|\mathbf{y}|) .
\end{gathered}
$$




\subsection{Boundary conditions.}

2.3.1. Boundary condition at zero. Using the Frobenius theory, e.g. [14, Theorem 4 p.165], the indicial equation at $r=0$ for (2.19) is,

$$
-\lambda^{2}+\lambda+\lim _{r \rightarrow 0} r^{2}\left(-\frac{\sigma^{2}(r)}{\mathfrak{c}^{2}(r)}+\mathrm{q}(r)\right)+\ell(\ell+1)=0 .
$$

Under (2.17), this simplifies to

$$
\lambda^{2}-\lambda-\ell(\ell+1)=0 \quad \Rightarrow \quad \lambda=-\ell \text { or } \lambda=\ell+1 .
$$

The regular solution at $r=0$ is given by the exponent $\lambda=\ell+1$, which explains the boundary condition (2.23). Under (2.17), for operator (2.19) (or generally other regular singular ODE with indicial roots of opposite signs), (2.23) can be replaced by the boundary condition

$$
\lim _{r \rightarrow 0} r \frac{d}{d r}\left(\frac{G_{\ell}(r)}{r}\right)=0,
$$

which also selects the regular solution at $r=0$. This can be seen as follows.

A generic solution in the neighborhood of zero is a linear combination of a function that decays in $r^{\ell+1}$ and one that blows up in $r^{-\ell}$; in particular, cf. [14, Theorem 4 p.165],

$$
w(r)=\mathrm{a}\left(r^{-\ell} h(r)+\mathrm{c}_{\ell}(\log r) r^{\ell+1} g(r)\right)+\mathrm{b} r^{\ell+1} g(r), \quad r>0,
$$

for some linear combination of constants a, b. Here, $c_{\ell}$ is a fixed constant (depending on $\ell$ ) and functions $g(r)$ and $h(r)$ are $C^{1}$ up to $r=0$ with

$$
g(0) \neq 0, \quad h(0) \neq 0 .
$$

The presence of the log term is due to the integral difference of the two indicial exponents, i.e. $(\ell+1)-(-\ell) \in \mathbb{N}$. We have

$$
\begin{gathered}
r\left(\frac{w}{r}\right)^{\prime}=\mathrm{a}\left((-\ell-1) r^{-\ell-1} h(r)+\mathrm{c} r^{\ell} g(r)+\ell \mathrm{c}(\log r) r^{\ell} g(r)\right)+\mathrm{b} \ell r^{\ell} g(r) \\
+\mathrm{a}\left(r^{-\ell} h^{\prime}(r)+\mathrm{c}(\log r) r^{\ell+1} g^{\prime}(r)\right)+\mathrm{b} r^{\ell+1} g^{\prime}(r) .
\end{gathered}
$$

$$
\begin{aligned}
\Longrightarrow & \lim _{r \rightarrow 0} r\left(\frac{w(r)}{r}\right)^{\prime}=\lim _{r \rightarrow 0} \mathrm{a}\left((-\ell-1) r^{-\ell-1} h(r)+r^{-\ell} h^{\prime}(r)\right), \quad \ell>0 ; \\
& \lim _{r \rightarrow 0} r\left(\frac{w(r)}{r}\right)^{\prime}=\mathrm{a}\left(\mathrm{c} g(0)+h^{\prime}(0)-\lim _{r \rightarrow 0} \frac{h(r)}{r}\right), \quad \ell=0 .
\end{aligned}
$$

It follows immediately that, for $w$ of the form (2.32) and for all $\ell \in \mathbb{N}$,

$$
\begin{aligned}
& \lim _{r \rightarrow 0} r\left(\frac{w(r)}{r}\right)^{\prime}<\infty \quad \Longrightarrow \quad \mathrm{a}=0 ; \\
& \text { and } \quad \mathrm{a}=0 \quad \Longleftrightarrow \lim _{r \rightarrow 0} r\left(\frac{w(r)}{r}\right)^{\prime}=0 .
\end{aligned}
$$


Remark 2.6. One can also consider a condition such that $\lim _{r \rightarrow 0} r^{2}(w / r)^{\prime}=0$, which also selects the regular family, i.e. forces $a=0$. The reasoning for $\ell>0$ is the same as above, due to the extra factor in $r$. For $\ell=0$, we have

$$
\begin{gathered}
r^{2}\left(\frac{w}{r}\right)^{\prime}=\mathrm{a}(-h(r)+\mathrm{crg}(r))+\mathrm{a}\left(r h^{\prime}(r)+\mathrm{c}(\log r) r^{2} g^{\prime}(r)\right)+\mathrm{b} r^{2} g^{\prime}(r) ; \\
\Longrightarrow \quad \lim _{r \rightarrow 0} r^{2}\left(\frac{w}{r}\right)^{\prime}=-\mathrm{a} h(0) .
\end{gathered}
$$

In using $h(0) \neq 0$, cf. (2.33), we also obtain, for $\ell=0$,

$$
\lim _{r \rightarrow 0} r^{2}\left(\frac{w}{r}\right)^{\prime}=0 \quad \Longrightarrow \quad \mathrm{a}=0 .
$$

In addition to being $\ell$-independent, the condition (2.31) is more natural and is simpler to implement in a discretization scheme, in the sense that it will lead to a Dirichlet-type boundary condition.

2.3.2. Outgoing boundary condition. For the numerical discretization on a finite domain, we need to replace the outgoing condition (2.24) by one on an artificial boundary. In particular in the case of the solar atmosphere with $\rho$ and $\mathfrak{c}$ extended according to the Atmo model on $\left(r_{\max }, \infty\right)$ (i.e., $\mathfrak{c}$ becomes constant and $\rho$ exponentially decaying in the atmosphere), we have an explicit expression for the Dirichlet-to-Neumann (DtN) coefficient, cf. [8, Section 4.1],

$$
\mathbf{Z}_{\mathrm{DtN}}^{\ell}(r):=-2 \mathrm{ik} \frac{\mathrm{W}_{-\frac{\mathrm{i} \alpha}{2 \mathrm{k}}, \ell+1 / 2}^{\prime}(-2 \mathrm{ik} r)}{\mathrm{W}_{-\frac{\mathrm{i} \alpha}{2 \mathrm{k}}, \ell+\frac{1}{2}}(-2 \mathrm{ik} r)},
$$

where $\mathrm{W}$ is the Whittaker's special function, cf. [8, 7], and $\mathrm{k}$ is defined by $(2.20)$ (Whittaker's functions are also discussed in [24] when considering exponential variation in density). The outgoing condition (2.24) is then replaced by

$$
\partial_{n} G_{\ell}=\mathbf{Z}_{\mathrm{DtN}}^{\ell} G_{\ell}
$$

Remark 2.7. Under more general assumptions of extensions, one does not have an explicit description of the DtN. However, if the extension still maintains the structure of the potential, as described in Assumptions 2.2 and 2.3, and since we work mode by mode, we can use the nonlocal radiation boundary condition and its high-frequency approximations, cf. subsection 5.3.

3. Numerical calculation of the Green's function. For the computation of the modal Green's function that solves (2.22), we consider the generic problem associated with a right-hand side $g$. By using the unknown $u=G / r$ and omitting the index $\ell$ for clarity, we thus consider, on the interval $\left[0, r_{\max }\right]$, the numerical calculation of the solution to

$$
\left\{\begin{array}{l}
-\left(r^{2} u^{\prime}(r)\right)^{\prime}+Q(r) u(r)=r g(r), \quad r \in\left(0, r_{\max }\right) ; \\
\lim _{r \rightarrow 0}\left(r u^{\prime}(r)\right)=0 ; \quad u^{\prime}\left(r_{\max }\right)=\left(-\frac{1}{r_{\max }}+\mathbf{Z}_{\bullet}\left(r_{\max }\right)\right) u\left(r_{\max }\right) .
\end{array}\right.
$$

We recall, from (2.18),

$$
\frac{Q(r)}{r^{2}}=-\frac{\sigma^{2}(r)}{\mathfrak{c}^{2}(r)}+\frac{\alpha^{2}(r)}{4}+\frac{\alpha^{\prime}(r)}{2}+\frac{\alpha(r)}{r}+\frac{\ell(\ell+1)}{r^{2}} .
$$


If the coefficient $\mathbf{Z}_{\mathrm{DtN}}^{\ell}(2.38)$ is used in the impedance condition at $r_{\max }$, then we have the exact outgoing solution (modulo the numerical discretization error). We will also investigate the effectiveness of approximate RBC coefficients, listed in (5.3) and (5.4).

3.1. First-order formulation for HDG discretization. In Proposition 2.4, the Green's function is constructed using the formula (2.26), which relies on the Wronskian, hence on the derivative of the solution to (3.1). This motivates the numerical implementation of the first-order formulation for the problem (3.1), where we introduce the new variables $w$ and $v$ such that,

$$
w:=r u \quad, \quad v:=r u^{\prime}, \quad \text { first-order variables. }
$$

With this choice of variables, the problem (3.1) is written as

$$
\left\{\begin{array}{l}
-r(r v(r))^{\prime}+Q(r) w(r)=r^{2} g(r), \quad r \in\left(0, r_{\max }\right) ; \\
r w^{\prime}(r)-w(r)=r v(r), \quad r \in\left(0, r_{\max }\right) ; \\
\lim _{r \rightarrow 0} v(r)=0, \quad v\left(r_{\max }\right)=\left(-\frac{1}{r_{\max }}+\mathbf{Z}_{\bullet}\left(r_{\max }\right)\right) w\left(r_{\max }\right) .
\end{array}\right.
$$

Here, the radiation condition in terms of $(w, v)$ is obtained by multiplying both sides of $u^{\prime}=\left(-\frac{1}{r_{\max }}+\mathbf{Z}_{\bullet}\right) u$ by $r_{\max }$, and replacing $u$ and $u^{\prime}$ by $w$ and $v$. Note that by solving the first-order problem, both $w$ and $v$ (i.e., $u$ and $u^{\prime}$ ) are obtained with the same accuracy while if we were to implement the second-order problem (3.1), the derivative of $u$ (needed for the Wronskian in (2.26)) would be retrieved with one order less accuracy than $u$.

For the implementation, we use the Hybridizable Discontinuous Galerkin (HDG, $[4,13,20])$ discretization method which, we believe, is the most appropriate. In other discretization methods such as finite elements or finite differences, the discretization leads to a linear system whose size is the number of degrees of freedom for all unknowns (here, $w$ and $v$ ). On the other hand, with the HDG method, the global linear system is only composed of the degrees of freedom of the numerical trace for one variable $(w)$, that is, those that are on the faces of the cells that constitute the discretized domain. Then, the volume solutions (for $w$ and $v$ ) are constructed locally via small (hence numerically cheap) linear systems.

The HDG method is specifically designed for first-order problems as it allows to maintain a small linear system compared to other discretizations, as observed by, e.g., $[13,22,10,15]$. The implementation follows two levels with first the global system for the numerical trace and then local systems for the volume solution. For our problem (3.4), these are detailed in our extended report, [6, Section 6].

3.2. Computation of the Green's function: Approach 1. The first, and most natural, approach for the computation of the Green's function $G_{\ell}$ is to solve numerically (2.22), that is, with a Dirac distribution as a right-hand side. Using the HDG first-order system, it amounts to Algorithm 3.1.

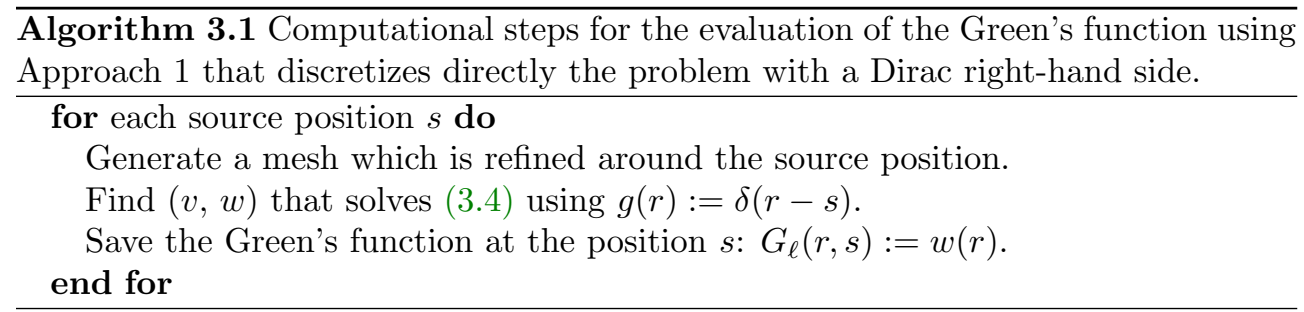


Here, for each resolution of the problem (3.4), i.e., for a fixed value of $s_{0} \in$ $\left(0, r_{\max }\right)$, one obtains the value of the Green's kernel $G_{\ell}\left(r, s_{0}\right)$ only on the vertical segment $\left[0, r_{\max }\right] \times s_{0}$, and its symmetric reflection across the diagonal $r=s_{0}$, see Figure 1(a). This means that to precisely evaluate the Green's function (i.e., using several sources), one needs to solve many problems. One can take advantage of the multiple right-hand sides features of direct solver such as Mumps ([3]) to calculate at the same time several solutions and obtain the value of $G_{\ell}$ on several vertical line segments (and hence their symmetric reflexion). This, however, requires the use of the same domain discretization for all of the right-hand sides, which is not appropriate with the discretization of a Dirac function, as we illustrate in subsection 3.4.

3.3. Computation of the Green's function: Approach 2. In the second approach, we make use of the formula (2.26) for the evaluation of the Green's function. Consider $0<r_{a}<r_{b} \leq r_{\max }$, we construct the two solutions, $\psi$ on $\left[0, r_{b}\right]$, and $\tilde{\psi}$ on $\left[r_{a}, r_{\max }\right]$, with each solving a boundary value problem, and we assemble the Green's function using (2.26). We detail the computational steps in Algorithm 3.2.

Algorithm 3.2 Computational steps for the evaluation of the Green's function using Approach 2 that uses Proposition 2.4. The complete Green's function is obtained from the solution of two boundary value problems.

Step 1a. Find $(v, w)$ that solves

$$
\begin{cases}-r(r v)^{\prime}+Q w=0, & \text { on }\left(0, r_{b}\right) \\ r w^{\prime}-w=r v, & \text { on }\left(0, r_{b}\right) ; \\ v(0)=0, \quad w\left(r_{b}\right)=1 . & \end{cases}
$$

Set $\psi:=w$ and $\psi^{\prime}:=v+w / r$.

Step 1b. Find $(v, w)$ that solves

$$
\begin{cases}-r(r v)^{\prime}+Q w=0, & \text { on }\left(r_{a}, r_{\max }\right) ; \\ r w^{\prime}-w=r v, & \text { on }\left(r_{a}, r_{\max }\right) ; \\ w\left(r_{a}\right)=1, \quad v\left(r_{\max }\right)= & \left(-\frac{1}{r_{\max }}+\mathbf{Z}_{\bullet}\right) w\left(r_{\max }\right) .\end{cases}
$$

Set $\tilde{\psi}:=w$ and $\tilde{\psi}^{\prime}:=v+w / r$.

Step 2. Using $\mathcal{W}(s)=\psi(s) \tilde{\psi}^{\prime}(s)-\psi^{\prime}(s) \tilde{\psi}(s)$, assemble the Green's function,

$$
G_{\ell}(r, s)=\frac{-\mathrm{H}(s-r) \psi(r) \tilde{\psi}(s)-\mathrm{H}(r-s) \tilde{\psi}(r) \psi(s)}{\mathcal{W}(s)} .
$$

Using this approach, from the solution of two boundary value problems, the Green's function $G_{\ell}(r, s)$ is obtained on the domain $\left[r_{a}, r_{b}\right] \times\left[r_{a}, r_{b}\right]$, as we illustrate in Figure 1. Approach 2 offers the following advantages compared to Approach 1.

- The Green's function is obtained for all pairs $(r, s)$ with $r, s \in\left[r_{a}, r_{b}\right]$ from the solutions of two problems, while with Approach 1, one problem only gives the Green's function at a fixed $s$.

- The functions $\psi$ and $\tilde{\psi}$ are regular on their corresponding domain of computation. They correspond to boundary value problems and they do not have a singular 


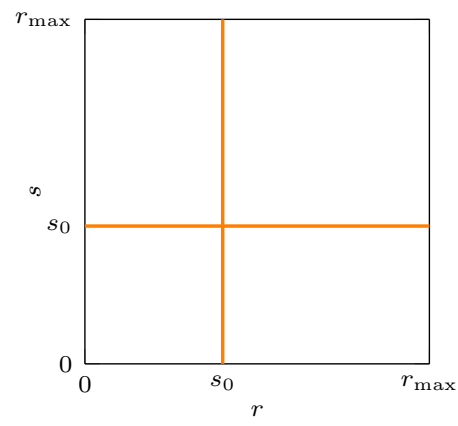

(a) Approach 1: the solution of one problem for a Dirac in $s_{0}$ only gives $G_{\ell}\left(r, s=s_{0}\right)$ and $G_{\ell}\left(r=s_{0}, s\right)$.

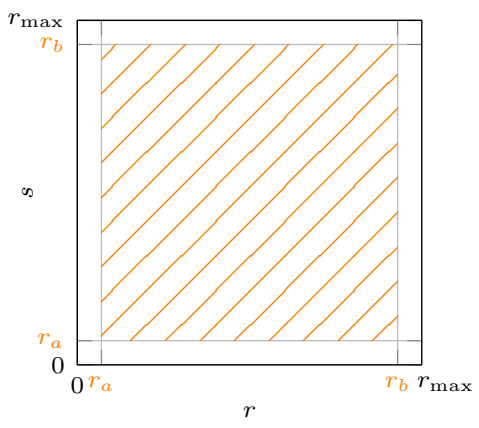

(b) Approach 2: from the solutions of two boundary value problems, $G_{\ell}(r, s)$ is obtained for any position between $r_{a}$ and $r_{b}$.

Fig. 1. Domain of definition (orange) of the modal Green's kernel for Approach 1 and 2, respectively given by Algorithms 3.1 and 3.2.

source. Because of this, one does not need to refine the mesh around the singularities of the source as in Approach 1, cf. subsection 3.4 and Remark 5.1.

Remark 3.1. The outgoing solution $\tilde{\psi}$ is a continuous solution on $[\epsilon, \infty)$ for arbitrarily small $\epsilon>0$, however it contains a singularity term that behaves like a constant $\times r^{-\ell}$ as $r \rightarrow 0$ for $\ell>0$. Therefore, the Dirichlet condition (3.6) cannot be extended to $r_{a}=0$ for $\ell>0$. This means $r_{a}$ is positive but can be arbitrarily small, cf. (3.13) for its numerical value in the experiment.

3.4. Numerical validation with the analytical solutions. To validate our numerical code based upon the HDG discretization (see [6, Section 6]), we compare the numerical and the analytical solutions, obtained by considering the first-order problem (3.4) with,

$$
\text { constant } \mathfrak{c} \text { and } \alpha \text { for } Q \text { in (3.2) (i.e., } \alpha^{\prime}=0 \text { ), }
$$$$
\text { a right-hand side } g=\delta(r-s) \text {, and the exact DtoN condition } \mathbf{Z}_{\mathrm{DtN}}^{\ell} \text {. }
$$

3.4.1. Analytical solution. Under (3.8), the exact solution $w_{\text {ref }}$ to (3.4) is given by (the details of which are given in our extended report [6, Section 7])

$$
w_{\mathrm{ref}}(r, s)=\frac{-\mathrm{H}(s-r) \psi(r) \tilde{\psi}(s)-\mathrm{H}(r-s) \tilde{\psi}(r) \psi(s)}{\mathcal{W}\{\psi(s), \tilde{\psi}(s)\}},
$$

where $\psi$ and $\tilde{\psi}$ are given in terms of the Whittaker's function $\mathrm{W}$ and of the regular Whittaker's (or Buchholtz) function M,

$$
\psi=\mathrm{M}_{\frac{\mathrm{i} \alpha}{2 \mathrm{k}}, \ell+1 / 2}(2 \mathrm{ik} r), \quad \tilde{\psi}=\mathrm{W}_{-\frac{\mathrm{i} \alpha}{2 \mathrm{k}}, \ell+1 / 2}(-2 \mathrm{ik} r) .
$$

We note that $\psi$ and $\tilde{\psi}$ can be chosen as constant multiples of the solutions of the boundary value problems. The Wronskian of $\psi$ and $\tilde{\psi}$ defined in (3.10) is given explicitly by $([6$, Section 7$])$,

$$
\mathcal{W}\{\psi(s), \tilde{\psi}(s)\}=2 \mathrm{i} \mathrm{k} \frac{\Gamma(2 \ell+2)}{\Gamma\left(1+\ell+\frac{\mathrm{i} \alpha}{2 \mathrm{k}}\right)}(-1)^{\ell+1},
$$

where $\Gamma$ is the (complex-valued) Gamma function. 
3.4.2. Numerical experiments. We choose constant parameters that are representative of the solar atmosphere. They are extracted from the Atmo model and are scaled such that,

$$
\mathfrak{c}=6.86 \times 10^{9} / R_{\odot}=9.87 \times 10^{-6} \mathrm{~s}^{-1} ; \quad \alpha=6663.62 .
$$

The (scaled) radius varies from 0 to 1.05 and we consider a fixed source at $s=1$. The comparison between the two approaches and the analytical solution is pictured in Figure 2, for a frequency of $6 \mathrm{mHz}$, with and without attenuation. Due to the fast oscillations of the solutions, we zoom in an interval around the source location. In Approach 2, we take the parameters

$$
r_{a}=10^{-25}, \quad r_{b}=r_{\max }=1.05,
$$

which give the interval of computation for $\psi$ and $\tilde{\psi}$, see (3.5) and (3.6).

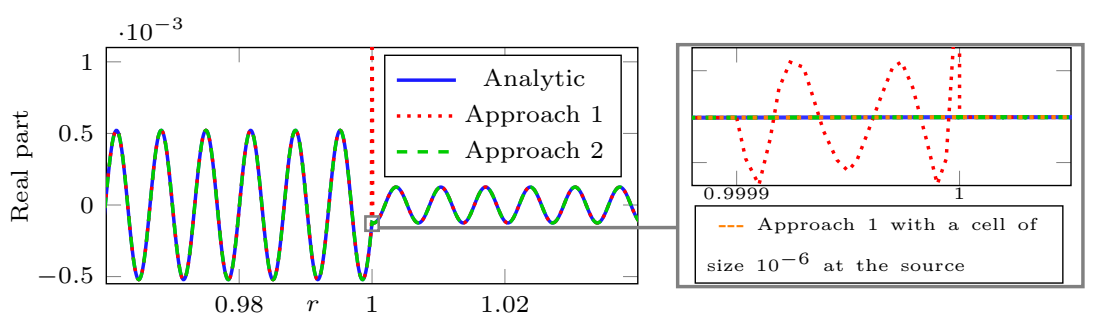

(a) Real part at $6 \mathrm{mHz}$ for $\ell=0$ without attenuation $(\gamma=0)$ and zoom near the source position $(r=1)$.
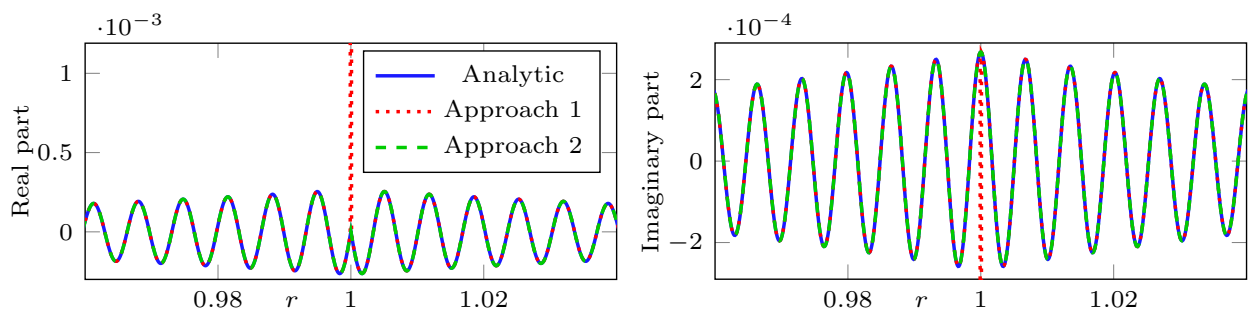

(b) Real and imaginary parts at $6 \mathrm{mHz}$ for $\ell=0$ with attenuation $\gamma /(2 \pi)=10^{-4} \mathrm{~Hz}$.

FIG. 2. Comparison of the solutions $w$ to the first-order conjugated problem (3.4) under (3.8) and (3.12) at $6 \mathrm{mHz}$ for mode $\ell=0$ without and with attenuation for a source located at $s=1$. The discretization uses a homogeneous mesh with elements of size $10^{-4}$ and polynomials of order 6 .

We observe that, away from the source location, the two approaches cannot be distinguished visually from the analytical solution. However, in the Approach 1, which discretizes the Dirac source function on the right-hand side, the singularity remains, leading to drastic inaccuracy, not only at the exact source location, but in the whole cell where it is contained, as highlighted in Figure 2(a), and here we need to divide the size of the cell by 100 to obtain an accurate solution. While the singularity is only in the real part when there is no attenuation $(\gamma=0)$, the inaccuracy also appears in the imaginary part in the general case $\gamma \neq 0$. This observation highlights a major difficulty of the naive discretization approach: the discretization of the source needs to be carefully addressed. This is even more important as, in helioseismology, one is mostly interested by the value of the solution at the source location (namely, $G_{\ell}(1,1)$ ). In order to overcome the issue, one needs to refine around the source 
position, as advocated in $[11,16,5]$. This can however be cumbersome when several sources must be taken into account, as the mesh should be updated for every source, or be over-refined everywhere. On the other hand, Approach 2 provides the accurate solution at the source position, without any need for refinement. Note also that, while $r_{a}$ must be different of 0 in Approach 2, here, an arbitrarily small value $\left(10^{-25}\right)$ works well.

In Table 1, we give the relative errors between the approaches for different frequencies and modes

$$
\mathcal{E}_{w}=\frac{\left\|w_{\mathrm{ref}}-w_{\bullet}\right\|_{L^{2}}}{\left\|w_{\mathrm{ref}}\right\|_{L^{2}}},
$$

where $w_{\bullet}$ is the computed solution using Approach 1 or 2. The error is computed on the whole interval (from 0 to $r_{\max }$ ). It confirms the accuracy of the numerical solutions, with a relative error smaller than $0.1 \%$. However, Approach 1 suffers from the inaccuracy near the source position, that leads to high error if the cell is not sufficiently refined.

TABLE 1

Relative error (3.14) between the analytical solution and the computations using Approach 1 or 2. The original mesh uses cells of size $10^{-4}$. The refined mesh is obtained by reducing the size of the cell (of the original mesh) that contains the source by 100. The error is multiplied by 100 to give the result in percent.

\begin{tabular}{|c|l||c|c||c|}
\hline $3 \mathrm{mHz}$ & $\begin{array}{c}\text { Approach 1 } \\
\text { (original mesh) }\end{array}$ & $\begin{array}{c}\text { Approach 1 } \\
\text { (refined mesh) }\end{array}$ & $\begin{array}{c}\text { Approach 2 } \\
\text { (original mesh) }\end{array}$ \\
\hline & $\ell=0, \gamma=0$ & $3.2 \times 10^{1 \%} \%$ & $2.4 \times 10^{-2} \%$ & $6.9 \times 10^{-3} \%$ \\
\hline & $\ell=100, \gamma=0$ & $3.1 \times 10^{1 \%} \%$ & $1.8 \times 10^{-2} \%$ & $6.6 \times 10^{-3} \%$ \\
\hline & $\ell=0, \gamma=10^{-4}$ & $2.1 \times 10^{2} \%$ & $1.8 \times 10^{-4} \%$ & $5.5 \times 10^{-5} \%$ \\
\hline & $\ell=100, \gamma=10^{-4}$ & $2.1 \times 10^{2} \%$ & $1.8 \times 10^{-4} \%$ & $5.5 \times 10^{-5} \%$ \\
\hline \hline $10 \mathrm{mHz}$ & & $\begin{array}{c}\text { Approach 1 } \\
\text { (original mesh) }\end{array}$ & $\begin{array}{c}\text { Approach 1 } \\
\text { (refined mesh) }\end{array}$ & $\begin{array}{c}\text { Approach 2 } \\
\text { (original mesh) }\end{array}$ \\
\hline & $\ell=0, \gamma=0$ & $1.1 \times 10^{1 \%} \%$ & $2.1 \times 10^{-2} \%$ & $9.9 \times 10^{-3} \%$ \\
\hline & $\ell=100, \gamma=0$ & $1.1 \times 10^{11 \%}$ & $9.4 \times 10^{-3} \%$ & $9.4 \times 10^{-3} \%$ \\
\hline & $\ell=0, \gamma=10^{-4}$ & $5.8 \times 10^{1 \%} \%$ & $6.3 \times 10^{-3} \%$ & $8.3 \times 10^{-3} \%$ \\
\hline & $\ell=100, \gamma=10^{-4}$ & $5.9 \times 10^{11 \%}$ & $6.3 \times 10^{-3} \%$ & $8.3 \times 10^{-3} \%$ \\
\hline
\end{tabular}

\section{Observables and data analysis in local helioseismology.}

4.1. Measured Observations and helioseismic products. In helioseismology, the measured data are time series representing line-of-sight Doppler velocities $\Phi_{\text {obs }}\left(\hat{\mathbf{r}}, t_{j}\right)$ at spatial points $\hat{\mathbf{r}}$ on the CCD (charge-coupled device) camera and at times $t_{j}$. Here, a spatial point is represented either by a two-dimensional vector $\hat{\mathbf{r}}$, or by its polar and azimuthal spherical angles $(\theta, \phi)$. These observables can come from interferometers on-board of satellites such as the Michelson Doppler Imager (MDI) or the Helioseismic and Magnetic Imager (HMI), or from ground-based telescopes such as the Global Oscillation Network Group (GONG). Upon performing a discrete Fourier transform, the time-series data can be turned into frequency observations $\Phi_{\text {obs }}(\hat{\mathbf{r}}, \omega)$. In practice, the signals can be filtered depending on the purpose of investigation, but we do not consider filtering in this paper.

Since the solar oscillations are driven by turbulent convection, the signals are realizations of random processes and analyzed by statistical methods. As the sources 
have zero mean, the expectation value of the observable $\mathbb{E}[\Phi(\hat{\mathbf{r}}, \omega)]=0$, which means that the data cannot be directly interpreted on the observed signal. Instead, timedistance helioseismology is based on the temporal cross-covariance $\mathcal{C}\left(\hat{\mathbf{r}}_{1}, \hat{\mathbf{r}}_{2}, t\right)$, between any two points, for a given $\Phi$,

$$
C\left(\hat{\mathbf{r}}_{1}, \hat{\mathbf{r}}_{2}, t\right)=\frac{1}{T} \int_{-T / 2}^{T / 2} \Phi\left(\hat{\mathbf{r}}_{1}, s\right) \Phi\left(\hat{\mathbf{r}}_{2}, t+s\right) \mathrm{d} s,
$$

where $T$ is the observation time. In the frequency domain, the definition of the crosscovariance is given by a multiplication,

$$
C\left(\hat{\mathbf{r}}_{1}, \hat{\mathbf{r}}_{2}, \omega\right)=\overline{\Phi\left(\hat{\mathbf{r}}_{1}, \omega\right)} \Phi\left(\hat{\mathbf{r}}_{2}, \omega\right) .
$$

The main quantities of interest in helioseismology are given below.

1. The power spectrum $\mathcal{P}$ represents the strength of the signal as a function of harmonic degrees and frequency. It is obtained from the harmonic spherical decomposition of the signal

$$
\mathcal{P}^{\ell, m}(\omega)=\mathbb{E}\left[\left|\Phi_{l}^{m}(\omega)\right|^{2}\right], \quad \text { with } \quad \Phi_{\ell}^{m}(\omega)=\int_{0}^{2 \pi} \int_{0}^{\pi} \Phi(\hat{\mathbf{r}}, \omega) \overline{\mathrm{Y}_{\ell}^{m}(\hat{\mathbf{r}})} \mathrm{d} \hat{\mathbf{r}} .
$$

2. The expectation value of the cross-covariance is used to represent the time-distance diagram, which visualizes how waves propagating through the solar interior are observed at the surface as a function of time and distance. It is given by

$$
\mathcal{C}\left(\hat{\mathbf{r}}_{1}, \hat{\mathbf{r}}_{2}, t\right):=\mathbb{E}\left[C\left(\hat{\mathbf{r}}_{1}, \hat{\mathbf{r}}_{2}, t\right)\right], \quad \mathcal{C}\left(\hat{\mathbf{r}}_{1}, \hat{\mathbf{r}}_{2}, \omega\right):=\mathbb{E}\left[C\left(\hat{\mathbf{r}}_{1}, \hat{\mathbf{r}}_{2}, \omega\right)\right] .
$$

4.2. Synthetic signals. The synthetic signals are created from solutions $\phi$ of the scalar wave equation (2.5) with a stochastic source $\mathfrak{s}$ on the right-hand side, i.e.

$$
\phi(\mathbf{r}, \omega)=\int \mathcal{G}(\mathbf{r}, \mathbf{s}, \omega) \mathfrak{s}(\mathbf{s}, \omega) \mathrm{d} \mathbf{s} .
$$

As the height (relative to the solar surface) variable plays a special role, we will often separate the vertical and horizontal variables and write $\mathbf{r}=(r, \hat{\mathbf{r}})$. We also denote by $\mathfrak{M}\left(\mathbf{s}, \mathbf{s}^{\prime}, \omega\right)$ the source covariance matrix

$$
\mathfrak{M}\left(\mathbf{s}, \mathbf{s}^{\prime}, \omega\right)=\mathbb{E}\left[\overline{\mathfrak{s}(\mathbf{s}, \omega)} \mathfrak{s}\left(\mathbf{s}^{\prime}, \omega\right)\right] .
$$

This is a distribution in $\mathbb{R}^{3} \times \mathbb{R}^{3}$ depending on the parameter $\omega$. Below, we write $<,>$ to denote the distribution pairing.

Assumption 4.1. We suppose that the observed signal is coming from the observation height $r_{\mathrm{obs}}$ such that the synthetic signal $\Phi$ is given by the trace of $\phi$ at $r=r_{\mathrm{obs}}$,

$$
\Phi(\hat{\mathbf{r}}, \omega)=\phi\left(r_{\text {obs }}, \hat{\mathbf{r}}, \omega\right) .
$$

Using Proposition 2.4 and Remark 2.5, it can be written in spherical harmonic expansion and in terms of the modal Green's function $\mathcal{G}_{\ell}$,

$$
\Phi(\hat{\mathbf{r}}, \omega)=\sum_{\ell=0}^{\infty} \sum_{m=-\ell}^{\ell} \int_{\mathbb{R}^{3}} \frac{\mathcal{G}_{\ell}\left(r_{\mathrm{obs}}, s ; \omega\right)}{r_{\mathrm{obs}} s} \mathrm{Y}_{\ell}^{m}(\hat{\mathbf{r}}) \overline{\mathrm{Y}_{\ell}^{m}(\hat{\mathbf{s}})} \mathfrak{s}(\mathbf{s}, \omega) \mathrm{d} \mathbf{s},
$$


with its spherical harmonic projection,

$$
\Phi_{\ell}^{m}(\omega)=\int_{\mathbb{R}^{3}} \frac{\mathcal{G}_{\ell}\left(r_{\mathrm{obs}}, s ; \omega\right)}{r_{\mathrm{obs}} s} \overline{\mathrm{Y}_{\ell}^{m}(\hat{\mathbf{s}})} \mathfrak{s}(\mathbf{s}, \omega) \mathrm{d} \mathbf{s} .
$$

In the above expressions, $\mathbf{s}=(s, \hat{\mathbf{s}})$ and $d \mathbf{s}=s^{2} \mathrm{~d} s \mathrm{~d} \hat{\mathbf{s}}$.

4.3. Synthetic helioseismic products. Using the notation $\mathfrak{M}$ in (4.6), we substitute expression (4.9) into the definition of the power spectrum (4.3), to obtain

$$
\mathcal{P}_{\ell}^{m}(\omega)=\left\langle\mathfrak{M}\left(\mathbf{s}, \mathbf{s}^{\prime}, \omega\right), \frac{\overline{\mathcal{G}_{\ell}\left(r_{\mathrm{obs}}, s ; \omega\right)}}{r_{\mathrm{obs}} s} \frac{\mathcal{G}_{\ell}\left(r_{\mathrm{obs}}, s^{\prime} ; \omega\right)}{r_{\mathrm{obs}} s^{\prime}} \mathrm{Y}_{\ell}^{m}(\hat{\mathbf{s}}) \overline{\mathrm{Y}_{\ell}^{m}\left(\widehat{\mathbf{s}^{\prime}}\right)}\right\rangle .
$$

To simplify the computation of the helioseismic observables, we consider the following assumption on the source, (cf. the Section 8 of our extended report [6]) for more details.

Assumption 4.2. The source $\mathfrak{s}$ is a random process with zero mean and covariance $\mathfrak{M}$,

$$
\mathfrak{M}\left(\mathbf{r}, \mathbf{r}^{\prime}, \omega\right)=\Pi(\omega) \mathcal{M}(r) \delta\left(\mathbf{r}-\mathbf{r}^{\prime}\right) .
$$

This form implies that the sources are spatially uncorrelated. Here, $\Pi$ is a function that is linked to the time correlation of the sources, and $\mathcal{M}(s)$ is a distribution in the variable $s$.

By the notation (4.11), we mean a distribution whose action is defined as, for a smooth compactly supported function $u\left(\mathbf{s} ; \mathbf{s}^{\prime}\right)=u\left(s, \hat{\mathbf{s}} ; s^{\prime}, \widehat{\mathbf{s}^{\prime}}\right) \in \mathcal{C}_{c}^{\infty}\left(\mathbb{R}^{3} \times \mathbb{R}^{3}\right)$,

$$
\left\langle\mathfrak{M}\left(\mathbf{s}, \mathbf{s}^{\prime} ; \omega\right), u\left(\mathbf{s}, \mathbf{s}^{\prime}\right)\right\rangle:=\Pi(\omega) \int_{\mathbb{S}^{2}}\left\langle\mathcal{M}(s), s^{4} u(s, \hat{\mathbf{s}} ; s, \hat{\mathbf{s}})\right\rangle d \hat{\mathbf{s}} .
$$

Remark 4.3. Assumption 4.2 is routinely employed in helioseismology, even if a more accurate description is possible, for example by replacing the delta function in (4.11) by a Gaussian with a given correlation length [18]. This is not a limitation for our approach. Specifically, we could work with the general formula (4.10). See also further discussion at the end of the subsection.

Under Assumption 4.2, using the orthonormality of the spherical harmonics, the expression of the power spectrum (4.10) simplifies to

$$
\mathcal{P}_{\ell}^{m}(\omega)=\frac{\Pi(\omega)}{r_{\text {obs }}^{2}}\left\langle\mathcal{M}(s), s^{2}\left|\mathcal{G}_{\ell}\left(r_{\mathrm{obs}}, s ; \omega\right)\right|^{2}\right\rangle .
$$

Convenient source. In order to simplify this expression, it is possible to find a convenient form of the function $\mathcal{M}$ such that the power spectrum directly relates to the Green's function for a source and receiver located at the observation height.

Assumption 4.4. $\mathcal{M}$ is given by the following distribution $\mathcal{M}_{\mathrm{eq}}$,

$$
\left\langle\mathcal{M}_{\mathrm{eq}}(s), u(s)\right\rangle:=\int_{0}^{\infty} \frac{\gamma(s)}{\rho(s) \mathfrak{c}^{2}(s) s^{2}} u(s) \mathrm{d} s, \quad u \in \mathcal{C}_{c}^{\infty}((0, \infty)) .
$$


Under Assumption 4.4, the power spectrum has the simplified form,

$$
\mathcal{P}_{\text {eq }}^{\ell}(\omega)=\frac{\Pi(\omega)}{2 \omega r_{\text {obs }}^{2}} \operatorname{Im}\left[\mathcal{G}_{\ell}\left(\mathrm{r}_{\mathrm{obs}}, \mathrm{r}_{\mathrm{obs}}, \omega\right)\right] .
$$

For derivation, see Appendix A. This relation between the power spectrum and the imaginary part of the Green's function is classic in geophysics under the hypothesis of energy equipartition (the energy current is identical in all directions, see, e.g., [27]). It has been used in helioseismology in [17] and reproduces the main features of the observed power spectrum. Morevoer, under this hypothesis, travel-time sensitivity kernels were computed in order to infer the meridional flow in the Sun, [19].

Single-depth assumptions. Another assumption used in helioseismology is that the sources are coming from a single depth denoted $r_{\mathrm{src}}$, [18]. In this case, we use the following assumption.

Assumption 4.5. The radial dependency $\mathcal{M}$, that we denote $\mathcal{M}_{\mathrm{sd}}$ is given by

$$
\mathcal{M}_{\mathrm{sd}}(s)=\delta\left(s-r_{\mathrm{src}}\right) \text {. }
$$

The expression of the power spectrum from (4.13) simplifies to,

$$
\mathcal{P}_{\mathrm{sd}}^{\ell}(\omega)=\frac{\Pi(\omega) r_{\mathrm{src}}^{2}}{r_{\mathrm{obs}}^{2}}\left|\mathcal{G}_{\ell}\left(r_{\mathrm{obs}}, r_{\mathrm{src}}, \omega\right)\right|^{2}
$$

As in the case of energy equipartition, the power spectrum is related to the Green's function at the observation height but the second point is now at the source location.

As the power spectrum is independant of $m$, the expectation value of the crosscovariance corresponds to the Legendre transform of the power spectrum [6, Section 8]

$$
\mathcal{C} \bullet\left(\hat{\mathbf{r}}_{1}, \hat{\mathbf{r}}_{2}, \omega\right)=\sum_{\ell=0}^{L_{\max }} \frac{2 \ell+1}{4 \pi} \mathcal{P}_{\bullet}^{\ell}(\omega) P_{\ell}\left(\cos \theta_{\hat{\mathbf{r}}_{1} \cdot \hat{\mathbf{r}}_{2}}\right) \quad \text { with } \bullet=\text { eq, sd. }
$$

The synthetic time-distance diagram is obtained from the inverse Fourier transform of the synthetic $\mathcal{C}_{\bullet}\left(\hat{\mathbf{r}}_{1}, \hat{\mathbf{r}}_{2}, \omega\right)$ with

$$
\mathcal{C} \bullet\left(\hat{\mathbf{r}}_{1}, \hat{\mathbf{r}}_{2}, t_{j}\right)=h_{\omega} \sum_{k=-N_{t} / 2}^{N_{t} / 2-1} \mathcal{C} \bullet\left(\hat{\mathbf{r}}_{1}, \hat{\mathbf{r}}_{2}, \omega\right) e^{-\mathrm{i} \omega t_{j}} ; \quad \text { with } \bullet=\text { eq, sd }
$$

In order to compute the power spectrum, one only needs the Green's function for a source location at $r_{\mathrm{obs}}$ and a receiver at $r_{\mathrm{obs}}$ or $r_{\mathrm{src}}$ depending on the assumption on the source. However, we made two major simplifications to obtain these formulae: 1. the observation height $r_{\mathrm{obs}}$ is a single height that is the same everywhere on the CCD. A more realistic assumption would require to integrate over depth depending on the opacity of the solar surface.

2. the sources are coming from a single depth $r_{\text {src }}$ (Assumption 4.5) or the energy is equipartitioned (Assumption 4.4). These are simplifying assumptions as the sources should come from a range of depths and the energy distribution in the Sun is complex. 


$$
\begin{array}{ll}
r \in[0,1.0008], & \text { interval for } \mathrm{S}+\mathrm{Atmo}, \text { up to } 550 \mathrm{~km} \text { above the surface. } \\
r \in[0,1.00365], & \text { interval for } \mathrm{S}+\mathrm{Val}-\mathrm{C}, \text { up to } 2.5 \mathrm{Mm} \text { above the surface. }
\end{array}
$$

5. Numerical experiments of helioseismic observables. In this section, we perform numerical experiments using inhomogeneous medium parameters that follow the solar profiles, using our numerical setup with the HDG method, validated above ${ }^{1}$ We use the Green's functions computed with Approach 2 to obtain the synthetic helioseismic quantities as defined in section 4 . It allows us to compare with observational data, to evaluate the performance of the boundary conditions and to illustrate the importance of the full Green's functions to generate synthetic data.

5.1. Solar Green's kernels for models $S+A t m o$ and $S+V a l-C$. The propagation of the scalar waves is governed by the medium wave speed $\mathfrak{c}$ and the inverse density scale height $\alpha$, together with its derivative $\alpha^{\prime}$. In the interior of the Sun, these are extracted from the model S of [12]. In the atmosphere, we consider two models: Atmo, where the velocity is smoothly extended to a constant and the density follows an exponential decay $([16,17])$; and the model Val-C of [28]. At the end of the model Val-C, we can further extend the model following the Atmo principles, to define the Val-C+Atmo model. The representations of the coefficients $\mathfrak{c}$ and $\alpha$ are given in Figure 3 for two solar profiles, S+Atmo and S+Val-C. The atmospheric profiles given by the model Val-C contains a drastic increase of both parameters $\mathfrak{c}$ and $\alpha$, while these quantities are constant in the Atmo model.

The interval for the computations ends as soon as the wave speed is constant and the density exponentially decaying (i.e., $\alpha$ is constant), such that

The attenuation is kept fixed to the value $\gamma / 2 \pi=20 \mu \mathrm{Hz}$ and the scaling function $\Pi$

\footnotetext{
${ }^{1}$ Our code, hawen, is written in Fortran90, and combines mpi and OpenMp parallelism, it is available at https://ffaucher.gitlab.io/hawen-website/. It is linked with the library Arb, [21] for the efficient computation of the special functions (i.e., the Whittaker's functions for the DtN coefficient).
} 


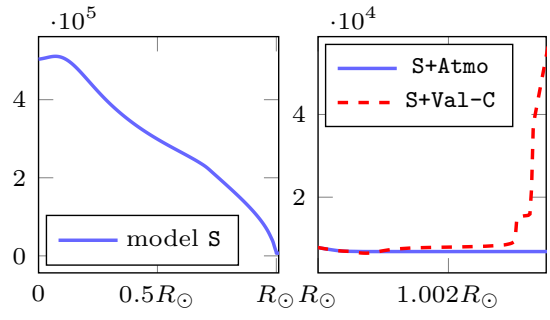

(a) Wave speed profile $\left(\mathrm{m} \mathrm{s}^{-1}\right)$.

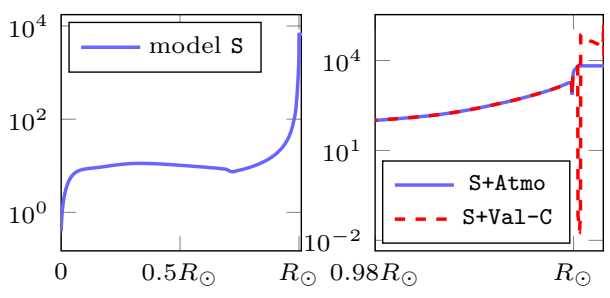

(b) profile for $\alpha=-\rho^{\prime} / \rho$.

FIG. 3. Profiles of the solar parameters, the model $S$ is used for the interior and is combined with the atmospheric models Atmo or Val-C.

is given by [17, Eq. (85)],

(5.2) $\Pi(\omega)=\left(1+\left(\frac{|\omega|-\omega_{0}}{\omega_{1}}\right)^{2}\right)^{-1}, \quad$ with $\frac{\omega_{0}}{2 \pi}=3.3 \mathrm{mHz}$ and $\frac{\omega_{1}}{2 \pi}=0.6 \mathrm{mHz}$.

We compare the modal Green's kernels for the two solar models, S+Atmo and S+Val-C in Figure 4 with $\mathbf{Z}_{\mathrm{DtN}}^{\ell}$ as boundary conditions (see subsection 5.3). Here, we use Approach 2 which gives access to the complete Green's kernel $G$ (of the conjugated problem) from two simulations. Then, $\mathcal{G}$ (for the original problem) is obtained after multiplying by the density, according to (2.13). We see that, for the two choices of models, the wavelength reduces when the waves are getting closer to the surface. Between the models $\mathrm{S}+\mathrm{Atmo}$ and $\mathrm{S}+\mathrm{Val}-\mathrm{C}$, there is a difference in amplitude and the patterns of the waves are also different. These are even more pronounced when we zoom near the surface (right of Figure 4), which is the area that is used to compute the following power spectrum.

Remark 5.1 (Computational cost of Approach 1). While Approach 2 gives the Green's function from two simulations, Approach 1 requires as many simulations as the number of sources, which must be at least a few thousand to later approximate the integral in (4.20). The multiple right-hand sides feature of direct solvers, such as MumPS [3], allows to mitigate the computational cost of having thousand of sources, however, as highlighted in Figure 2, one must also refine the mesh near the sources to obtain accurate solutions, consequently increasing the number of degrees of freedom. This need for an extra refinement was also emphasized in [17] in order to obtain accurate helioseismic observables. This is in particular crucial for $\mathcal{P}_{\text {eq }}$, (4.15) that relies on $\mathcal{G}_{\ell}(r, r)$. We illustrate the two approaches in Figure 5, where we have 4000 sources and we need to use cells of size $10^{-7}$ near the sources to ensure the accuracy of Approach 1. In our experiments, we consequently observe an increase in the computational time by a factor from 15 (for 4000 sources) to 30 (for 10000 sources) with Approach 1. Moreover, Approach 1 requires a large amount of memory to store the solutions for all sources while Approach 2 only requires the two simulations (this is not to be neglected when considering the number of frequencies and modes one needs to compute).

5.2. Computational experiments of power spectrum. Using the computational Approach 2, we obtain the full Green's functions and instantly have access to the power spectrum of subsection 4.2, associated with any heights for the source and the receiver. In our computations, we use frequencies from 1 to $12 \mathrm{mHz}$ and modes 


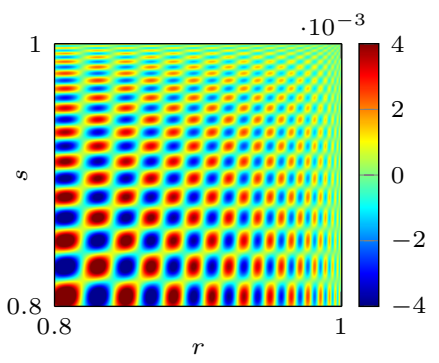

(a) $G_{\ell}(r, s)$, model S+Atmo.

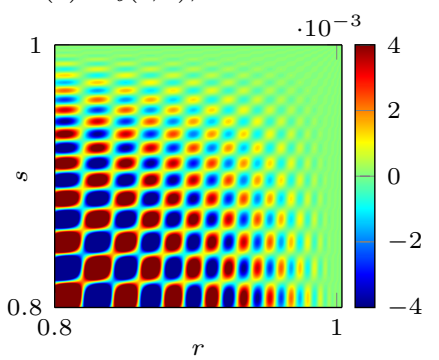

(c) $G_{\ell}(r, s)$, model S+Val-C.

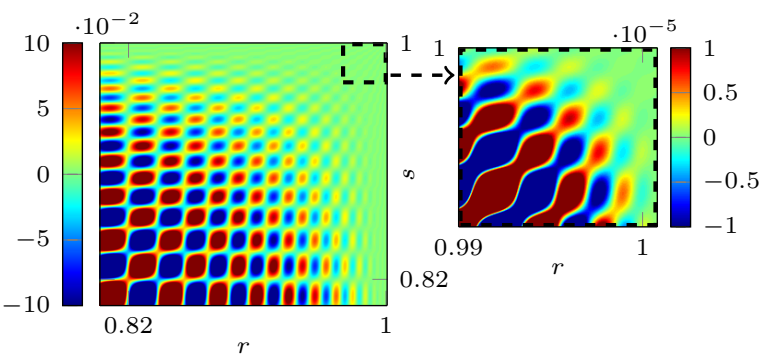

(b) $\mathcal{G}_{\ell}(r, s)$ model S+Atmo.

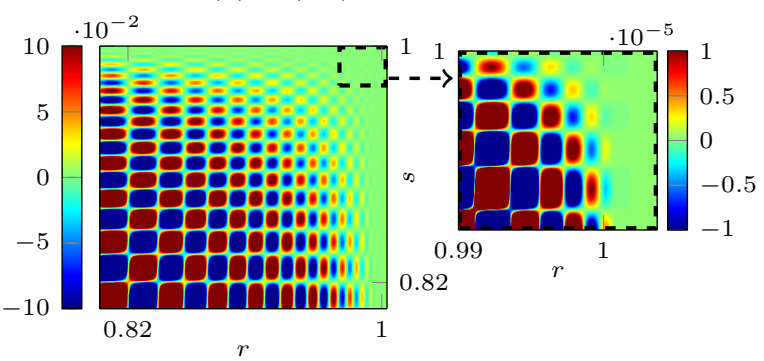

(d) $\mathcal{G}_{\ell}(r, s)$, model S+Val-C.

FIG. 4. Imaginary part of the Solar modal Green's functions at $7 \mathrm{mHz}$ for mode $\ell=100$ for the models $S+A$ tmo (top) and $S+V a l-C$ (bottom). The solution to the original problem and the conjugated one, respectively $\mathcal{G}_{\ell}$ and $G_{\ell}$, are related by (2.13).

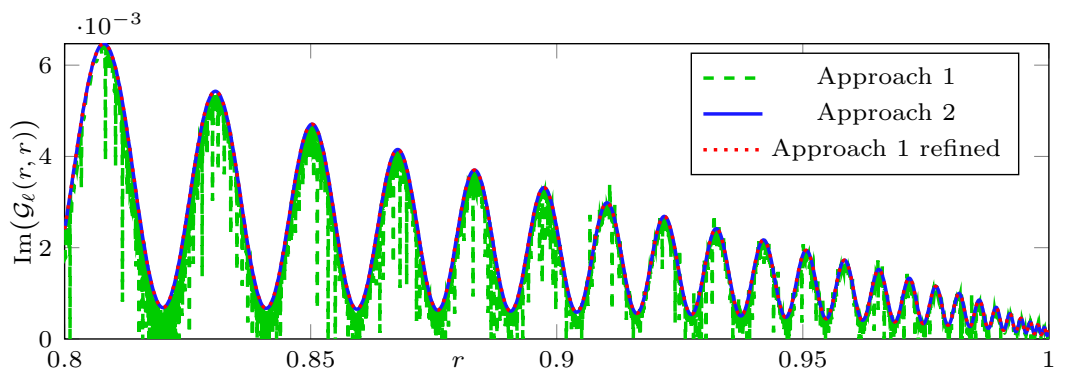

FIG. 5. Comparison of the Green's function for model $S+A$ tmo at frequency $7 \mathrm{mHz}$ and mode $\ell=100$. While Approach 2 depends on two simulations, Approach 1 needs one simulation for each of the 4000 sources. In Approach 1 with the refined mesh, the cell at the positions of the sources is of size $10^{-7}$.

from $\ell=0$ to 1000 , with a constant attenuation $\gamma /(2 \pi)=20 \mu \mathrm{Hz}$. Note that, contrary to the existing literature, our computation uses the exact Dirichlet-to-Neumann map condition $\mathbf{Z}_{\mathrm{DtN}}^{\ell}$ given in (2.38). In solar applications, the source depth $r_{\mathrm{src}}$ corresponds to the location where the waves are excited and is generally considered to be a bit below the surface (usually a few hundreds kilometers). The observation height depends on the instrument and is usually located slighly above the surface (up to 500 $\mathrm{km}$ ). It might appear as a small interval as $1 \mathrm{Mm}$ represents approximately $1 \times 10^{-3}$ in the scaled axis, nonetheless, we shall see that it leads to drastic differences. 


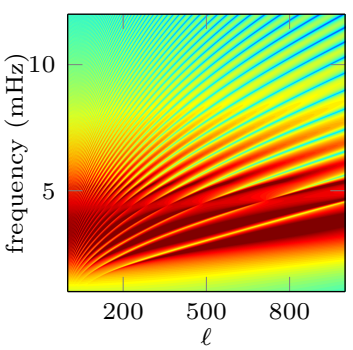

(a) $\mathcal{P}_{\text {sd }}$ for a source at $-1 \mathrm{Mm}$ of the surface.

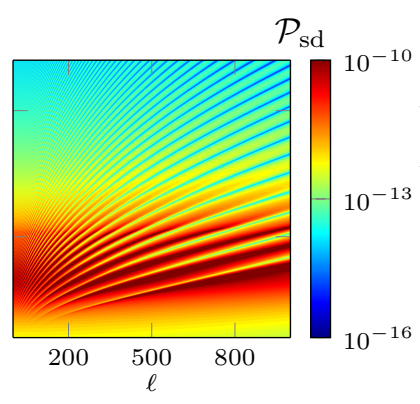

(b) $\mathcal{P}_{\text {sd }}$ for a source at $-340 \mathrm{~km}$ of the surface.

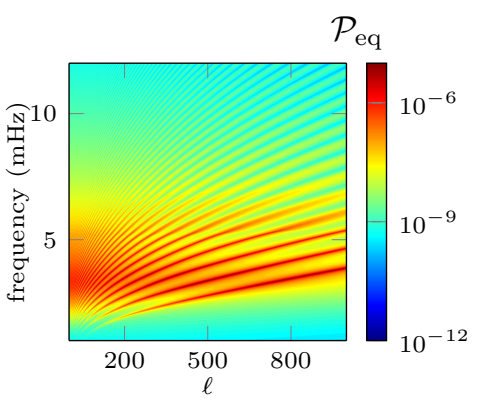

(c) $\mathcal{P}_{\text {eq }}$, the source is at the surface.

Fig. 6. Power spectra $\mathcal{P}_{\text {eq }}$ and $\mathcal{P}_{\mathrm{sd}},(4.15)$ and (4.17), for a receiver positioned at the surface, using the model S+Atmo with $\mathbf{Z}_{D t N}^{\ell}$ for $R B C$. For $\mathcal{P}_{\mathrm{sd}}$, the different formation source heights are given relative to the solar surface $R_{\odot}$.

To highlight the differences, we picture a line of the spectra, for a fixed mode $\ell=200$, in Figures 7 and 8 . We show the results for different source heights using the original or normalized spectra. We see that there is a strong difference in amplitudes depending on the formulation, in part due to the different scaling in (4.15) and (4.17). Comparing between the different source heights for $\mathcal{P}_{\text {sd }}$, we can visually note the differences in the shape of the ridges in Figure 6. This is further confirmed by the section at mode $\ell=200$ in Figures 7 and 8. At low frequencies, not all of the peaks appear, for instance the ones between 4 and $5 \mathrm{mHz}$ in Figure 8. If the sources of excitation are too deep then some modes are not excited leading to the absence of ridge in the power spectrum. At high frequencies, the spectra are very different depending on the assumption on the source covariance: here, both the amplitude and the phase are affected, cf. Figure 8.

5.2.2. Influence of the formation height. We represent the power spectra $\mathcal{P}_{\text {sd }}$ (4.17) for two solar models with a constant source height in Figure 9. We vary the position of the receiver, $r_{\text {obs }}$, which corresponds to the observation height and can be different depending on the instrument. We show the normalized section at mode $\ell=200$ in Figure 10.

We observe strong differences in both amplitude and phase depending on the positions of the observation height. While the two models, S+Atmo and S+Val-C corresponds well at low frequency, see Figure 10, the high-frequency profiles are totally different, with sharp or smooth peaks. In addition, we observe lines of reduced power with S+Val-C in the complete spectrum of Figure 9.

5.3. Efficiency of the radiation boundary conditions (RBC). Contrary to previous work, we analyze the performance of the RBCs in the context of helioseis- 

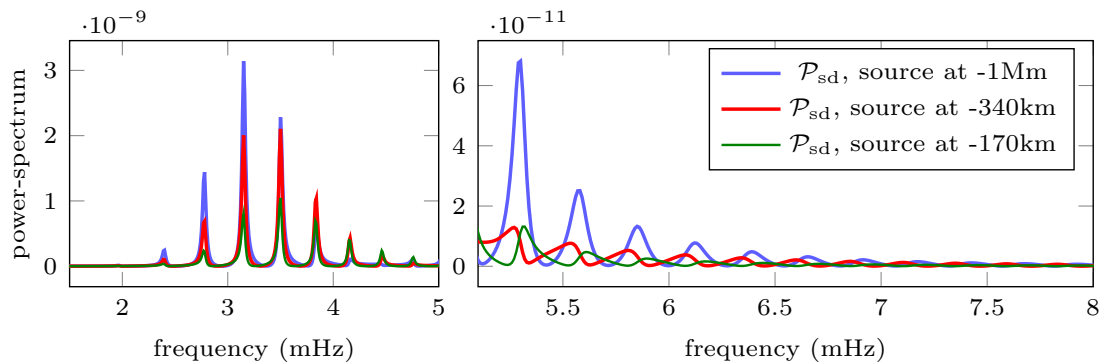

FIG. 7. Comparison of the line of the power spectrum $\mathcal{P}_{\mathrm{sd}}$, (4.17), at a fixed mode $\ell=200$, for different source heights, given relative to the solar surface. The receiver is positioned at the solar surface and the computations use model $S+A$ tmo with $\mathbf{Z}_{D t N}^{\ell}$ for $R B C$. We separate the low and high frequencies, which are visualized on different scales.
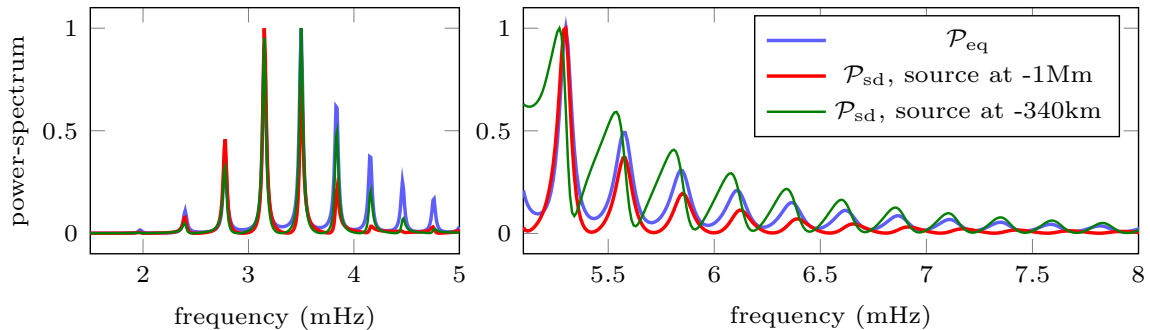

FIG. 8. Comparison of the line of the power spectra at a fixed mode $\ell=200$, for different source heights, given relative to the solar surface. The receiver is positioned at the solar surface and the computations use model $S+A$ tmo with $\mathbf{Z}_{D t N}^{\ell}$ for $R B C$. The low and high frequencies are independently normalized.

mic products, and using the exact DtN as the reference solution. We have in mind discretization in dimensions higher than one, where it is necessary to select a RBC that does not depend on the mode $\ell$, i.e., one that does not contain a tangential differential operator. We first recall the nonlocal RBC from $[5,8]$,

$$
\mathbf{Z}_{\text {nonlocal }}^{\ell}(r)=\mathrm{i}\left(\frac{\sigma^{2}(r)}{\mathfrak{c}^{2}(r)}-\mathrm{q}(r)-\frac{\ell(\ell+1)}{r^{2}}\right)^{1 / 2}
$$

Different high-frequency approximations of $\mathbf{Z}_{\text {nonlocal }}^{\ell}$ have been given in $[5,8]$ and we investigate the performance of (in the notation of [8]):

$$
\begin{array}{ll}
\mathbf{Z}_{\mathrm{SAI}-0}=\mathrm{ik}\left(1-\frac{\alpha_{\infty}}{r_{\max }} \frac{1}{\mathrm{k}^{2}}\right)^{1 / 2}, & \mathbf{Z}_{\mathrm{S}-\mathrm{HF}-0}=\mathrm{ik}, \\
\mathbf{Z}_{\mathrm{S}-\mathrm{HF}-1 \mathrm{a}}=\mathrm{ik}-\frac{\mathrm{i}}{2 \mathrm{k}} \frac{1}{r_{\max }} \alpha_{\infty}, & \mathbf{Z}_{\mathrm{A}-\mathrm{RBC}-1}:=\frac{1}{r_{\max }}+\mathrm{ik} ; \\
\mathbf{Z}_{\mathrm{A}-\mathrm{HF}-1}^{\ell}=\mathrm{i} \frac{\sigma}{\mathfrak{c}_{\infty}}+\frac{\mathfrak{c}_{\infty}}{2 \mathrm{i} \sigma}\left(\frac{\ell(\ell+1)}{r_{\max }^{2}}+\frac{\alpha_{\infty}}{r_{\max }}+\frac{\alpha_{\infty}^{2}}{4}\right) .
\end{array}
$$

The exponent $\ell$ indicates that the condition depends on the mode. Note that, while we provide comparisons for helioseismic quantities below, the performance of the conditions is evaluated analytically in our extended report, [6, Section 5]. 


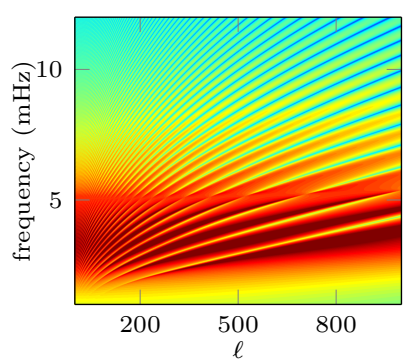

(a) $(0 \mathrm{~km},-695 \mathrm{~km})$

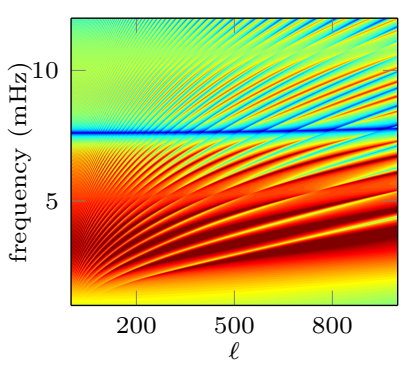

(e) $(0 \mathrm{~km},-695 \mathrm{~km})$

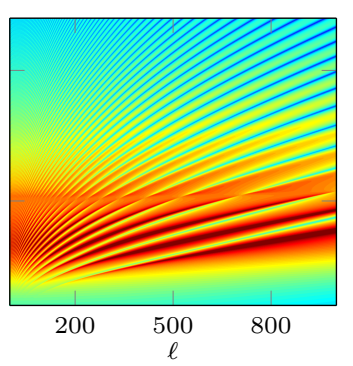

(b) $(170 \mathrm{~km},-695 \mathrm{~km})$

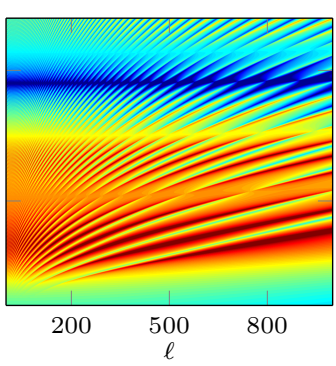

(f) $(170 \mathrm{~km},-695 \mathrm{~km})$

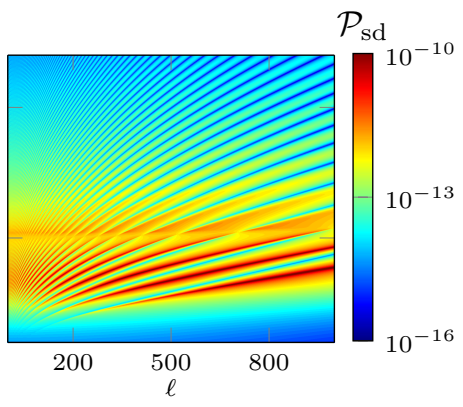

(c) $(340 \mathrm{~km},-695 \mathrm{~km}) \mathcal{P}_{\mathrm{sd}}$

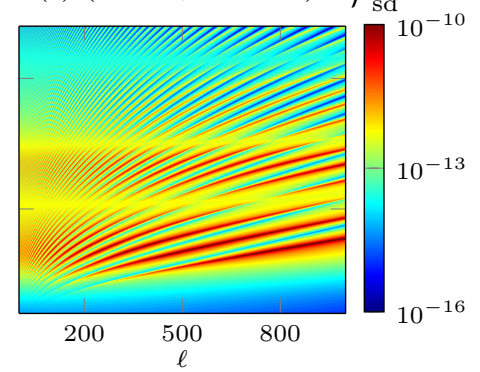

(g) $(340 \mathrm{~km},-695 \mathrm{~km})$

FIG. 9. Power spectrum $\mathcal{P}_{\text {sd }}$, (4.17), at different formation heights on a logarithmic scale. It uses the solar model S+Atmo on top and S+Val-C at the bottom. Each spectrum corresponds to a position of a receiver and a source, which are indicated in parenthesis and given relative to the solar surface $R_{\odot}$, i.e., the source is fixed at $695 \mathrm{~km}$ below the surface.
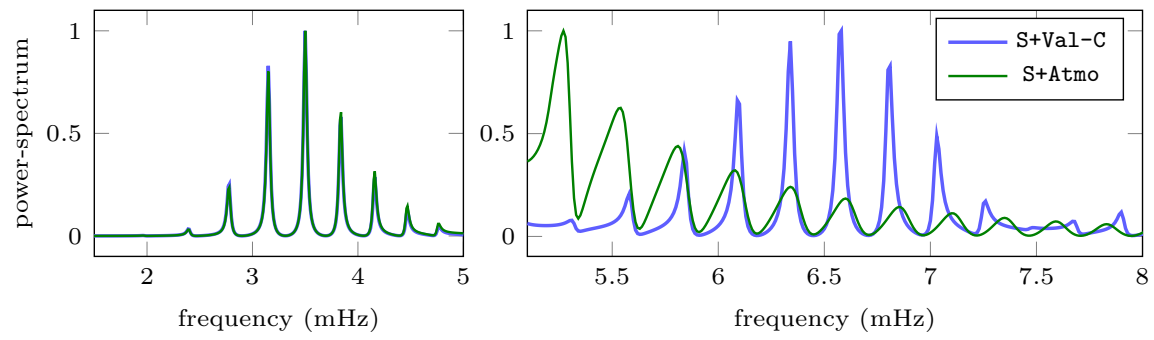

FIG. 10. Comparison of the line of the power spectrum, (4.17), at a fixed mode $\ell=200$, for a receiver and a source at respectively $340 \mathrm{~km}$ and $-695 \mathrm{~km}$ of the solar surface. The low and high frequencies are independently normalized.

5.3.1. Radiation boundary conditions for the power spectrum. We use the solar model S+Atmo, where the reference solution is given by the solution using the exact Dirichlet-to-Neumann map condition $\mathbf{Z}_{\mathrm{DtN}}^{\ell}$. We compute the power spectrum $\mathcal{P}_{\text {eq }}$ of (4.15) for $r_{\text {obs }}=1$, with different boundary conditions, and evaluate the performance with the relative error

$$
\mathfrak{e}_{\mathbf{Z}_{\bullet}}^{\mathcal{P}}(\omega, \ell)=\frac{\left\|\mathcal{P}_{\text {eq }, \mathbf{Z}_{\bullet}}^{\ell}(\omega)-\mathcal{P}_{\text {eq, }, \mathbf{Z}_{\mathrm{DtN}}^{\ell}}^{\ell}(\omega)\right\|}{\left\|\mathcal{P}_{\text {eq, }, \mathbf{Z}_{\mathrm{DtN}}^{\ell}}^{\ell}(\omega)\right\|},
$$


where the index $\mathbf{Z}$ • indicates the choice of condition with $\mathbf{Z}_{\mathrm{DtN}}^{\ell}$ giving the reference one. The relative errors are shown in Figure 11.

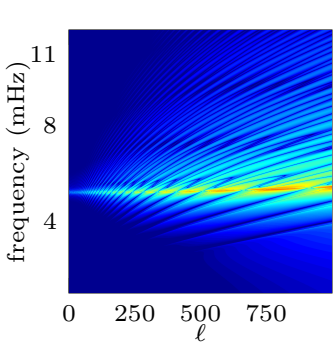

(a) $\mathfrak{e}^{\mathcal{P}} \mathbf{Z}_{\text {nonlocal }}^{\ell}$

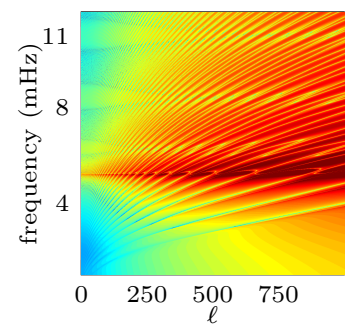

(e) $\mathfrak{e}_{\mathbf{Z}_{\text {S-HF-0 }}^{\mathcal{P}}}$

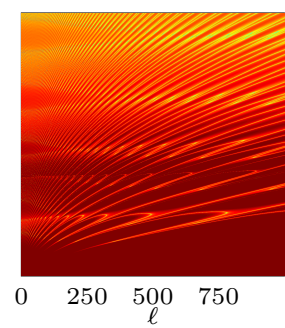

(b) $\mathfrak{e}_{\mathbf{Z}_{\mathrm{A}-\mathrm{HF}-1}^{\ell}}^{\mathcal{P}}$

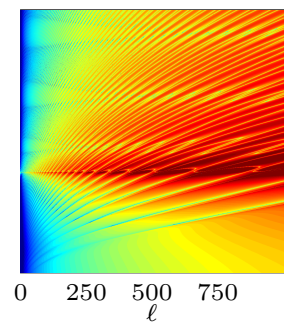

(f) $\mathfrak{e}_{\mathbf{Z}_{\text {S-HF-1a }}}$

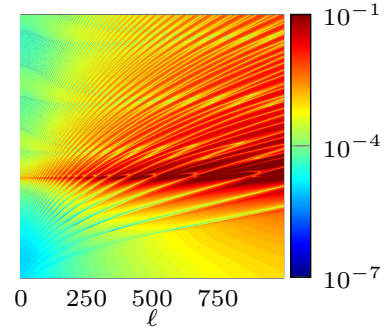

(c) $\mathfrak{e}_{\mathbf{Z}_{\mathrm{A}-\mathrm{BBC}-1}^{\mathcal{P}}}$

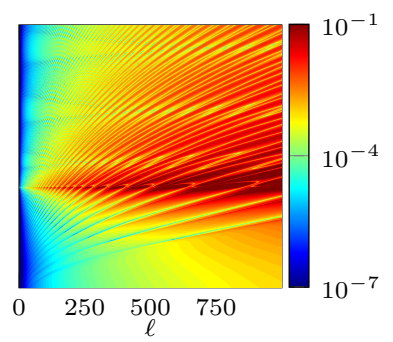

$(\mathrm{g}) \mathfrak{e}_{\mathbf{Z}_{\text {SAI-0 }}^{\mathcal{P}}}^{\mathcal{P}}$

FIG. 11. Relative error (5.5) for the power spectrum associated with the model S+Atmo depending on the choice of radiation boundary condition.

We see that the nonlocal boundary condition provides the most accurate results, and that the maximum error is on a line that corresponds to the cut-off frequency, in particular for high-degree modes. On the other hand, $\mathbf{Z}_{\mathrm{A} \text {-HF-1 }}$ gives the worst results, while all other conditions give very similar errors, with a slight advantage towards $\mathbf{Z}_{\mathrm{SAI}-0}$ and $\mathbf{Z}_{\mathrm{S}-\mathrm{HF}-1 \mathrm{a}}$. We further provide the means of the relative errors:

$$
\begin{aligned}
& \operatorname{mean}\left(\mathfrak{e}_{\mathbf{Z}_{\text {nonlocal }}^{\ell}}^{\mathcal{P}}\right) \quad=1 \times 10^{-5}, \quad \operatorname{mean}\left(\mathfrak{e}_{\mathbf{Z}_{\mathrm{A}-\mathrm{FF}-1}^{\ell}}^{\mathcal{P}}\right)=4.53 \times 10^{-1}, \\
& \operatorname{mean}\left(\mathfrak{e}_{\mathbf{Z}_{\text {S-HF- } 0}^{\mathcal{P}}}\right)=9.26 \times 10^{-3}, \quad \operatorname{mean}\left(\mathfrak{e}_{\mathbf{Z}_{\text {A-RBC-1 }}}^{\mathcal{P}}\right)=9.28 \times 10^{-3} \text {, } \\
& \operatorname{mean}\left(\mathfrak{e}_{\mathbf{Z}_{\text {S-HF-1a }}^{\mathcal{P}}}^{\mathcal{P}}\right)=9.10 \times 10^{-3}, \quad \operatorname{mean}\left(\mathfrak{e}_{\mathbf{Z}_{\text {SAI- }}}^{\mathcal{P}}\right)=9.10 \times 10^{-3} .
\end{aligned}
$$

5.3.2. Radiation boundary conditions for time-distance diagram. We compute the time-distance diagram using the exact $\mathbf{Z}_{\mathrm{DtN}}^{\ell}$ and compare with two highfrequency approximations of the nonlocal RBC coefficient: $\mathbf{Z}_{\mathrm{S}-\mathrm{HF}-1 \mathrm{a}}$ and $\mathbf{Z}_{\mathrm{A}-\mathrm{HF}-1}^{\ell}$. The first approximation is obtained with an expansion in $\mathrm{k}^{-1}$ while the latter in terms of $\sigma^{-1}$, cf. [8]. In addition, $\mathbf{Z}_{\mathrm{A}-\mathrm{HF}-1}^{\ell}$ depends on $\ell$ but not $\mathbf{Z}_{\mathrm{S}-\mathrm{HF}-1 \mathrm{a}}$, meaning that the latter is much more convenient to implement in 3D. Figure 12 shows the time-distance diagram for $\mathcal{C}_{\text {surf }}(\theta, t)$ where $\theta=\hat{\mathbf{r}}_{1} \cdot \hat{\mathbf{r}}_{2}$ is the angle between the two observation points $\hat{\mathbf{r}}_{1}$ and $\hat{\mathbf{r}}_{2}$. We first compute $\mathcal{C}_{\text {surf }}(\theta, \omega)$ using (4.18) with a frequency $h_{\omega}=5 \mu \mathrm{Hz}$ and then apply the inverse Fourier transform using (4.19) with $N_{t}=2^{15}$. We show the section at $\theta=30^{\circ}$ for the different RBC, together with the difference with respect to the reference (using $\mathbf{Z}_{\mathrm{DtN}}^{\ell}$ ) solution. 


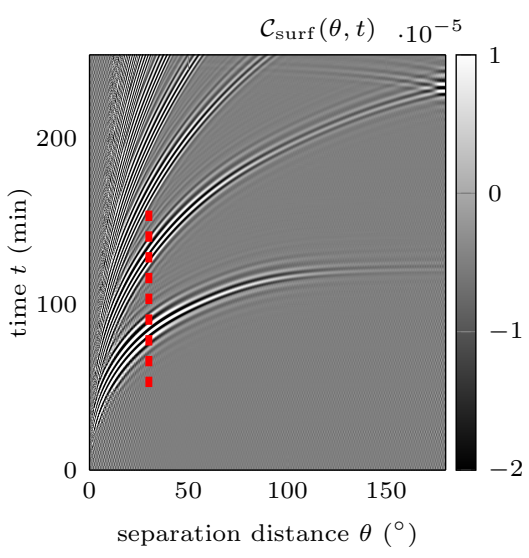

(a) Time-distance diagram with the DtN boundary condition.
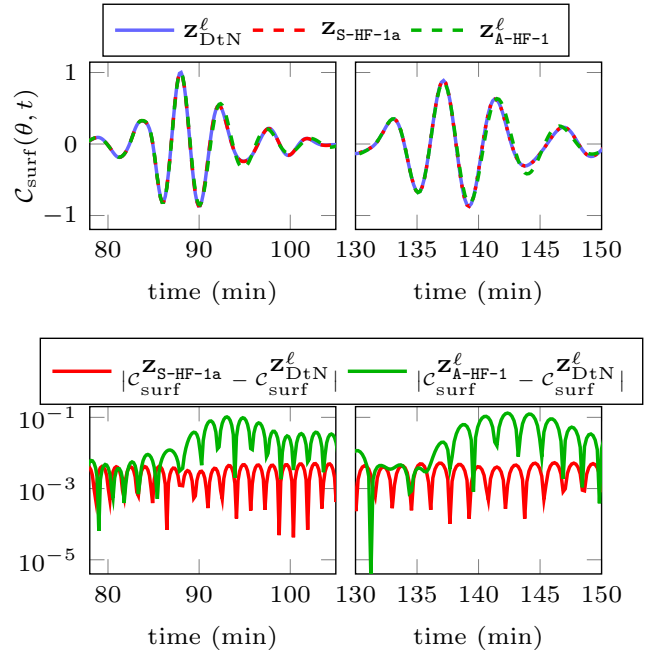

(b) Line at $\theta=30^{\circ}$ (top) and difference between the boundary conditions (bottom)

FIG. 12. Time-distance diagram representing the expectation value of the cross-covariance as a function of separation distance and time: two-dimensional diagram and the section for $\theta=30^{\circ}$, indicated by the red dashed lines on the left panel.

We see that in Figure 12(a), the time-distance diagram shows different skips corresponding to waves going directly from $\hat{\mathbf{r}}_{1}$ to $\hat{\mathbf{r}}_{2}$, or being reflected at the surface once or several times. We can visually observe the difference in the solutions between $\mathbf{Z}_{\mathrm{DtN}}^{\ell}$ and $\mathbf{Z}_{\mathrm{A}-\mathrm{HF}-1}^{\ell}$, which differ in phase and amplitude. The condition $\mathbf{Z}_{\mathrm{S}-\mathrm{HF}-1 \mathrm{a}}$ gives the best result (that is, the closest to $\mathbf{Z}_{\mathrm{DtN}}^{\ell}$ ) and we have between 2 to 3 orders of magnitude difference in the accuracy. Thus, working with the wavenumber $k$ leads to an improved performance compared to using $\sigma / \mathfrak{c}_{\infty}$.

6. Conclusion. In this work, we have proposed a two-step algorithm to compute efficiently and accurately the full outgoing modal Green's kernel for the scalar wave equation in local helioseismology under spherical symmetry. The full Green's kernel enables the computations of more realistic synthetic observables with varying observation heights, leading to drastic differences. It gives a convenient framework to study the origin of sources of excitation in the Sun. With its low cost, the algorithm not only opens up new applications that are based on better synthetic representations of the observations (e.g., with integrated quantities), but also has direct implications in inverse problems for the determination of the solar interior. Finally, our comparison of RBC also paves the way towards three-dimensional discretizations, by singling out the condition $\mathbf{Z}_{\mathrm{S}-\mathrm{HF}-1 \mathrm{a}}$ for its independence of the harmonic mode, and its high accuracy in approximating the outgoing solution.

Appendix A. Power spectrum under Assumptions 4.2 and 4.4.

Here, we show that the power spectrum takes the form given in (4.15), under Assumptions 4.2 and 4.4. To find this relation, let us write

$$
\overline{L_{\ell}} \overline{G_{\ell}\left(r_{1}, r\right)}=\delta\left(r_{1}-r\right), \quad L_{\ell} G_{\ell}\left(r_{2}, r\right)=\delta\left(r_{2}-r\right) .
$$

We then multiply the first equation by $G_{\ell}\left(r_{2}, r\right)$, the second one by $\overline{G_{\ell}\left(r_{1}, r\right)}$ and 
integrate to obtain

$$
\int_{0}^{\infty}\left(\overline{L_{\ell}} \overline{G_{\ell}\left(r_{1}, r\right)} G_{\ell}\left(r_{2}, r\right)-L_{\ell} G_{\ell}\left(r_{2}, r\right) \overline{G_{\ell}\left(r_{1}, r\right)}\right) \mathrm{d} r=G_{\ell}\left(r_{2}, r_{1}\right)-\overline{G_{\ell}\left(r_{2}, r_{1}\right)} .
$$

Canceling out the identical terms leads to

$$
\begin{aligned}
G_{\ell}\left(r_{2}, r_{1}\right) & -\overline{G_{\ell}\left(r_{2}, r_{1}\right)}=4 \mathrm{i} \omega \int_{0}^{\infty} \frac{\gamma(r)}{\mathfrak{c}^{2}(r)} \overline{G_{\ell}\left(r_{1}, r\right)} G_{\ell}\left(r_{2}, r\right) \mathrm{d} r \\
& +\int_{0}^{\infty}\left(-\frac{\mathrm{d}^{2}}{\mathrm{~d} r^{2}} \overline{G_{\ell}\left(r_{1}, r\right)} G_{\ell}\left(r_{2}, r\right)+\overline{G_{\ell}\left(r_{1}, r\right)} \frac{\mathrm{d}^{2}}{\mathrm{~d} r^{2}} G_{\ell}\left(r_{2}, r\right)\right) \mathrm{d} r .
\end{aligned}
$$

Integrating by part twice, the second line of the equation is equal to zero and thus

$$
\operatorname{Im}\left[G_{\ell}\left(r_{2}, r_{1}\right)\right]=2 \omega \int_{0}^{\infty} \frac{\gamma(r)}{\mathfrak{c}^{2}(r)} \overline{G_{\ell}\left(r_{1}, r\right)} G_{\ell}\left(r_{2}, r\right) \mathrm{d} r .
$$

Using (2.28) to come back to the Green's function of the original problem, we obtain

$$
\operatorname{Im}\left[\mathcal{G}_{\ell}\left(r_{2}, r_{1}\right)\right]=2 \omega \int_{0}^{\infty} \frac{\gamma(r)}{\rho(r) \mathfrak{c}^{2}(r)} \overline{\mathcal{G}_{\ell}\left(r_{1}, r\right)} \mathcal{G}_{\ell}\left(r_{2}, r\right) \mathrm{d} r .
$$

Identifying this expression with (4.10), we see that the choice of this distribution in (4.14) leads to the simplified expression for the power spectrum (4.15).

Acknowledgments. The authors thank the reviewers for their valuable comments that have helped improve the manuscript. This work is supported by the Inria associated-team Ants (Advanced Numerical meThods for helioSeismology) between project-team Inria Magique 3D and the Max Planck Institute for Solar System Research in Göttingen. FF is funded by the Austrian Science Fund (FWF) under the Lise Meitner fellowship M 2791-N. The numerical experiments have been performed as part of the GENCI resource allocation project AP010411013.

\section{REFERENCES}

[1] A. D. Agaltsov, T. Hohage, and R. G. Novikov, Global uniqueness in a passive inverse problem of helioseismology, Inverse Problems, (2020).

[2] S. Agmon, M. Klein, ET AL., Analyticity properties in scattering and spectral theory for Schrödinger operators with long-range radial potentials, Duke Mathematical Journal, 68 (1992), pp. 337-399.

[3] P. R. Amestoy, I. S. Duff, J.-Y. L'Excellent, and J. Koster, A fully asynchronous multifrontal solver using distributed dynamic scheduling, SIAM Journal on Matrix Analysis and Applications, 23 (2001), pp. 15-41.

[4] D. N. Arnold, F. Brezzi, B. Cockburn, And L. D. Marini, Unified analysis of discontinuous galerkin methods for elliptic problems, SIAM journal on numerical analysis, 39 (2002), pp. 1749-1779.

[5] H. Barucq, J. Chabassier, M. Duruflé, L. Gizon, and M. Leguèbe, Atmospheric radiation boundary conditions for the Helmholtz equation, ESAIM: Mathematical Modelling and Numerical Analysis, 52 (2018), pp. 945-964.

[6] H. Barucq, F. Faucher, D. Fournier, L. Gizon, and H. Pham, Efficient computation of the modal outgoing Green's kernel for the scalar wave equation in helioseismology, Research Report RR-9338, Inria Bordeaux Sud-Ouest ; Magique 3D ; Max-Planck Institute for Solar System Research, April 2020, https://hal.archives-ouvertes.fr/hal-02544701.

[7] H. BarucQ, F. FAudher, AND H. Pham, Outgoing solutions to the scalar wave equation in helioseismology, Research Report RR-9280, Inria Bordeaux Sud-Ouest ; Project-Team Magique3D, August 2019, https://hal.archives-ouvertes.fr/hal-02168467. 
[8] H. BARucq, F. FAucher, AND H. Pham, Outgoing solutions and radiation boundary conditions for the ideal atmospheric scalar wave equation in helioseismology, ESAIM: Mathematical Modelling and Numerical Analysis, 54 (2020), pp. 1111-1138.

[9] P. G. Bergmann, The wave equation in a medium with a variable index of refraction, The Journal of the Acoustical Society of America, 17 (1946), pp. 329-333.

[10] M. Bonnasse-Gahot, H. Calandra, J. Diaz, and S. LAnteri, Hybridizable discontinuous galerkin method for the 2-d frequency-domain elastic wave equations, Geophysical Journal International, 213 (2017), pp. 637-659.

[11] J. Chabassier And M. Durufle, High Order Finite Element Method for solving Convected Helmholtz equation in radial and axisymmetric domains. Application to Helioseismology, Research Report RR-8893, Inria Bordeaux Sud-Ouest, Mar. 2016, https://hal.inria.fr/ hal-01295077.

[12] J. Christensen-Dalsganrd, W. Däppen, S. Ajukov, E. Anderson, H. Antia, S. Basu, V. Baturin, G. Berthomieu, B. Chaboyer, S. Chitre, et Al., The current state of solar modeling, Science, 272 (1996), pp. 1286-1292.

[13] B. Cockburn, J. Gopalakrishnan, And R. LAzarov, Unified hybridization of discontinuous galerkin, mixed, and continuous galerkin methods for second order elliptic problems, SIAM Journal on Numerical Analysis, 47 (2009), pp. 1319-1365.

[14] E. A. Coddington, An introduction to ordinary differential equations, Dover Publisher, 1961.

[15] F. FAUChER AND O. SchERZER, Adjoint-state method for Hybridizable Discontinuous Galerkin discretization: application to the inverse acoustic wave problem, Computer Methods in Applied Mechanics and Engineering, 372 (2020), https://doi.org/10.1016/j.cma.2020.113406.

[16] D. Fournier, M. Leguèbe, C. S. Hanson, L. Gizon, H. Barucq, J. Chabassier, and M. Duruflé, Atmospheric-radiation boundary conditions for high-frequency waves in time-distance helioseismology, Astronomy \& Astrophysics, 608 (2017), p. A109.

[17] L. Gizon, H. Barucq, M. Duruflé, C. S. Hanson, M. Leguèbe, A. C. Birch, J. Chabassier, D. Fournier, T. Hohage, And E. Papini, Computational helioseismology in the frequency domain: acoustic waves in axisymmetric solar models with flows, Astronomy \& Astrophysics, 600 (2017), p. A35.

[18] L. Gizon And A. Birch, Time-distance helioseismology: the forward problem for random distributed sources, The Astrophysical Journal, 571 (2002), p. 966.

[19] L. Gizon, R. H. Cameron, M. Pourabdian, Z.-C. Liang, D. Fournier, A. C. Birch, And C. S. HAnson, Meridional flow in the Sun's convection zone is a single cell in each hemisphere, Science, 368 (2020), pp. 1469-1472.

[20] R. GRIESMAIER AND P. MONK, Error analysis for a hybridizable discontinuous galerkin method for the helmholtz equation, Journal of Scientific Computing, 49 (2011), pp. 291-310.

[21] F. JohAnsson, Arb: efficient arbitrary-precision midpoint-radius interval arithmetic, IEEE Transactions on Computers, 66 (2017), pp. 1281-1292.

[22] R. M. Kirby, S. J. Sherwin, And B. Cockburn, To cg or to hdg: a comparative study, Journal of Scientific Computing, 51 (2012), pp. 183-212.

[23] D. Lynden-Bell AND J. Ostriker, On the stability of differentially rotating bodies, Monthly Notices of the Royal Astronomical Society, 136 (1967), pp. 293-310.

[24] P. Martin, Acoustic scattering by inhomogeneous spheres, The Journal of the Acoustical Society of America, 111 (2002), pp. 2013-2018.

[25] P. A. Martin, Acoustic scattering by inhomogeneous obstacles, SIAM Journal on Applied Mathematics, 64 (2003), pp. 297-308.

[26] K. Nagashima, B. Löptien, L. Gizon, A. C. Birch, R. Cameron, S. Couvidat, S. DANIlOVIC, B. FleCK, AND R. STEIN, Interpreting the helioseismic and magnetic imager (HMI) multi-height velocity measurements, Solar Physics, 289 (2014), pp. 3457-3481.

[27] R. Snieder, M. Miyazawa, E. Slob, I. Vasconcelos, and K. Wapenaar, A Comparison of Strategies for Seismic Interferometry, Surveys in Geophysics, 30 (2009), pp. 503-523.

[28] J. E. Vernazza, E. H. Avrett, AND R. Loeser, Structure of the solar chromosphere. IIIModels of the EUV brightness components of the quiet-sun, The Astrophysical Journal Supplement Series, 45 (1981), pp. 635-725.

[29] D. YAnG, Modeling experiments in helioseismic holography, PhD thesis, The Georg-AugustUniversität Göttingen, 2018. 